\title{
The Conservation and Convergence of Two Finite Difference Schemes for KdV Equations with Initial and Boundary Value Conditions
}

\author{
Jinye Shen, Xuping Wang and Zhi-zhong Sun* \\ School of Mathematics, Southeast University, Nanjing 210096, Jiangsu, China
}

Received 8 March 2019; Accepted (in revised version) 28 August 2019

\begin{abstract}
Korteweg-de Vries equation is a nonlinear evolutionary partial differential equation that is of third order in space. For the approximation to this equation with the initial and boundary value conditions using the finite difference method, the difficulty is how to construct matched finite difference schemes at all the inner grid points. In this paper, two finite difference schemes are constructed for the problem. The accuracy is second-order in time and first-order in space. The first scheme is a two-level nonlinear implicit finite difference scheme and the second one is a threelevel linearized finite difference scheme. The Browder fixed point theorem is used to prove the existence of the nonlinear implicit finite difference scheme. The conservation, boundedness, stability, convergence of these schemes are discussed and analyzed by the energy method together with other techniques. The two-level nonlinear finite difference scheme is proved to be unconditionally convergent and the three-level linearized one is proved to be conditionally convergent. Some numerical examples illustrate the efficiency of the proposed finite difference schemes.
\end{abstract}

AMS subject classifications: 65M06, 65M12, 65M15

Key words: Nonlinear Korteweg-de Veries equation, difference scheme, existence, conservation, boundedness, convergence.

\section{Introduction}

In recent years, with most complex phenomenon appearing, nonlinear evolutionary equations [7, 23, 24] have become more and more important tool to describe them. Especially, Korteweg-de Vries (KdV) type equation has been widely applied in physics, mathematics, biophysics, which originated from modeling the shallow water surface height of solitary dispersive waves. In 1877, Boussinesq firstly discovered the KdV equation. After about twenty years, Korteweg and his $\mathrm{PhD}$ student Gustav de Vries mathematically reintroduced the KdV equation [5, 14].

*Corresponding author. Email addresses: goldleaf0811@sina.com (J. Y. Shen), seuMathWxp@139.com (X. P. Wang), zzsun@seu.edu.cn (Z. Z. Sun) 
The simplest form of KdV equation is as follows:

$$
u_{t}+\epsilon u u_{x}+\mu u_{x x x}=0,
$$

where $\epsilon, \mu$ are given constants.

There are many works on KdV equation. Kamruzzaman used the Kudryashov method to find the exact travelling wave solutions transmutable to the solitary wave solutions of the ubiquitous unsteady Korteweg-de Vries equation and applied the $\exp (-\phi(\xi))$-expansion method to construct the exact travelling wave solutions for nonlinear evolution equations $[12,13]$. About the numerical approximation of the onedimensional simplified KdV equation, many researchers have obtained abundant results by finite difference method, spectral method and finite volume method. For example, Vliegenthart discussed some explicit finite difference schemes for solving the initialvalue problem of KdV equation and presented dissipative difference schemes which had the effect of eliminating high wave number components. However, these schemes were conditionally stable [22]. The authors of [15] proposed a Legendre pseudo-spectral method for the KdV equation with nonperiodic boundary condition and analyzed the convergence for linear-KdV equation. Pazoto got a one order fully-implicit numerical scheme based on this asymptotic behavior of the solution of the generalized Kortewegde Vries equation (GKdV with $p=4$ ) and got convergece in $L_{4}$ norm [16]. Dougalis constructed a fully discrete Galerkin method with high order of accuracy for the numerical solution of the periodic initial-value problem for KdV equation. But they needed certain mild restriction on the space mesh length and the time step [9]. Alisamii et al. introduced a hybridized discontinuous Galerkin method to deal with nonlinear KdV type equations. As for the time stepping, they used the backward difference formula. However, there was no analysis of convergence in this research [2]. Yan proposed three conservative finite volume element schemes based on the discrete variational derivative method [26], but there was no analysis of convergence. Winebery developed an implicit-stepping scheme for $\mathrm{KdV}$ equation in temporal direction and spectral methods in space [25]. However, there was a restriction on the size of the time step when they applied predictor-corrector method to retain the full accuracy of the scheme. In addition, Bosco presented a finite difference method for the integration of the KdV equation with periodic boundary conditions on irregular grid. The method is shown to be superconvergent, which only took place on grids with an odd number of points per period [10]. Zhu constructed a difference scheme with a higher-order discrete invariant for the periodic KdV equation [27]. Djidjeli et al. proposed two numerical methods for the solution of the third- and fifth-order KdV equations. The first method was derived using central differences to replace the space derivative with predictor-corrector time-stepping and the second method by linearizing the implicit corrector scheme in which the solution was then found by solving a linear algebraic system at each time step. They proved the stability of these schemes [8]. And Qu and Wang had presented an alternating segment explicit-implicit difference scheme and proved the stability of this scheme by the analysis of linearization procedure [17]. Nonetheless, there were lack of the convergence analysis. 
To the best of our knowledge, the nonlinear term makes it difficult to study. Vliegenthart set up a difference scheme to solve the initial-value problem of KdV equation without $u_{x x x}(x, t)$ term and analyzed the period solution [22]. Recently, Atouani and Omrani [4] had designed two kinds of finite difference schemes of the Rosenau-Korteweg de Vries equation in 2D with periodic boundary problem and proved existence, conservative, stability and convergence of these schemes. What's more, Holden et al. [11] had considered finite difference scheme of KdV equation with decaying case on the full line and the periodic case. Furthermore, if the initial data $\left.u\right|_{t=0}=u_{0}$ is high regularity, the solution of the scheme is convergent to a classical solution and if the regularity of the initial data is less, then the scheme is convergent in some weak sense. Amorim and Figueira [3] had studied the convergence of a semi-discrete finite difference method for KdV equation by introducing a small term $h \Delta_{h}^{2} u$. It was known that the problem will be simplified by introducing a small term or mixed derivative.

In this article, we construct a two-level nonlinear and a three-level linearized finite difference scheme for the initial-boundary value problem of KdV equation:

$$
\begin{array}{ll}
u_{t}+\gamma u u_{x}+u_{x x x}=0, & 0<x<L, \quad 0<t \leq T, \\
u(x, 0)=\varphi(x), & 0 \leq x \leq L, \\
u(0, t)=0, \quad u(L, t)=0, \quad u_{x}(L, t)=0, \quad 0 \leq t \leq T,
\end{array}
$$

where $\varphi(0)=\varphi(L)=\varphi^{\prime}(L)=0, \gamma$ is a constant.

Our main novelty of this work lies in finding numerically and theoretically convergent difference schemes for the non-periodic boundary value problem of the KdV equation.

The rest of this paper is organized as follows. Some notations are introduced and three auxiliary lemmas are presented in Section 2. The conservation of the solution of the problem (1.1a)-(1.1c) is presented. A two-level nonlinear implicit finite difference scheme is constructed and the existence, boundedness and unconditional convergence are discussed in Section 3. A three-level linearized difference scheme is established and the same properties as in section 3 are presented by energy method in Section 4 . In the section 5 , we will give some numerical simulation using the finite difference schemes above. The paper ends with a brief conclusion in Section 6 .

\section{Preliminaries}

In this section, we mainly introduce some notations and preparatory lemmas. Take two positive integers $m$ and $n$. Let $h=L / m, x_{i}=i h(0 \leq i \leq m), \tau=T / n$, $t_{k}=k \tau(0 \leq k \leq n) ; \Omega_{h}=\left\{x_{i} \mid 0 \leq i \leq m\right\}, \Omega_{\tau}=\left\{t_{k} \mid 0 \leq k \leq n\right\}$. Let $\mathcal{U}_{h}=\left\{u \mid u=\left(u_{0}, \cdots, u_{m}\right)\right\}, \stackrel{\mathcal{U}}{h}_{h}=\left\{u \mid u \in \mathcal{U}_{h}, u_{0}=u_{m}=0\right\}$ be two grid function spaces defined on $\Omega_{h}$. For any grid function $u \in \mathcal{U}_{h}$, we introduce the following nota- 
tions:

$$
\begin{array}{ll}
\delta_{x} u_{i-\frac{1}{2}}=\frac{1}{h}\left(u_{i}-u_{i-1}\right), & 1 \leq i \leq m ; \\
\delta_{x}^{2} u_{i}=\frac{1}{h^{2}}\left(u_{i-1}-2 u_{i}+u_{i+1}\right), & 1 \leq i \leq m-1 ; \\
\delta_{x}^{3} u_{i+\frac{1}{2}}=\delta_{x}^{2}\left(\delta_{x} u_{i+\frac{1}{2}}\right), & 1 \leq i \leq m-2 ; \\
\Delta_{x} u_{i}=\frac{1}{2 h}\left(u_{i+1}-u_{i-1}\right), & 1 \leq i \leq m-1 ; \\
\psi(v, u)_{i}=\frac{1}{3}\left[v_{i} \Delta_{x} u_{i}+\Delta_{x}(v u)_{i}\right], & 1 \leq i \leq m-1 .
\end{array}
$$

For $w=\left\{w^{k} \mid 0 \leq k \leq n\right\}$ defined on $\Omega_{\tau}$, we introduce the following notations:

$$
\begin{aligned}
& w^{k-\frac{1}{2}}=\frac{1}{2}\left(w^{k}+w^{k-1}\right), \quad \delta_{t} w^{k-\frac{1}{2}}=\frac{1}{\tau}\left(w^{k}-w^{k-1}\right), \quad 1 \leq k \leq n, \\
& w^{\bar{k}}=\frac{1}{2}\left(w^{k+1}+w^{k-1}\right), \quad \Delta_{t} w^{k}=\frac{1}{2 \tau}\left(w^{k+1}-w^{k-1}\right), \quad 1 \leq k \leq n-1 .
\end{aligned}
$$

For any $u, v \in \mathcal{U}_{h}$, the inner products and norms are defined by

$$
\begin{aligned}
& (u, v)=h\left(\frac{1}{2} u_{0} v_{0}+\sum_{i=1}^{m-1} u_{i} v_{i}+\frac{1}{2} u_{m} v_{m}\right), \\
& \left(\delta_{x} u, \delta_{x} v\right)=h \sum_{i=0}^{m-1}\left(\delta_{x} u_{i+\frac{1}{2}}\right)\left(\delta_{x} v_{i+\frac{1}{2}}\right), \\
& \|u\|=\sqrt{(u, u)}, \quad|u|_{1}=\sqrt{\left(\delta_{x} u, \delta_{x} u\right)} .
\end{aligned}
$$

Lemma 2.1 ([19]). Suppose $v \in \mathcal{U}_{h}, w \in \stackrel{\mathcal{U}}{h}_{h}$. we have

$$
(\psi(v, w), w)=0
$$

Lemma 2.2 ([20]). For any $u \in \dot{\mathcal{U}}_{h}$, there is

$$
\|u\|_{\infty} \leq \frac{\sqrt{L}}{2}|u|_{1}, \quad|u|_{1} \leq \frac{2}{h}\|u\| .
$$

Lemma 2.3. Suppose $w \in \dot{\mathcal{U}}_{h}$. Then it holds that

$$
\begin{aligned}
& h \sum_{i=1}^{m-2}\left(\delta_{x}^{2} \delta_{x} w_{i+\frac{1}{2}}\right) w_{i}+\frac{1}{h}\left(\delta_{x} w_{m-\frac{3}{2}}-3 \delta_{x} w_{m-\frac{1}{2}}\right) w_{m-1} \\
= & \frac{1}{2} h^{2} \sum_{i=1}^{m-1}\left(\delta_{x}^{2} w_{i}\right)^{2}+\frac{1}{2}\left(\delta_{x} w_{\frac{1}{2}}\right)^{2}+\frac{3}{2}\left(\delta_{x} w_{m-\frac{1}{2}}\right)^{2} .
\end{aligned}
$$


Convergence of Finite Difference Schemes for KdV Equations

Proof. Set

$$
v_{m}=0, \quad v_{i}=\delta_{x} w_{i+\frac{1}{2}}, \quad i=m-1, m-2, \cdots, 0 .
$$

Then

$$
\begin{aligned}
& h \sum_{i=1}^{m-2}\left(\delta_{x}^{2} \delta_{x} w_{i+\frac{1}{2}}\right) w_{i}+\frac{1}{h}\left(\delta_{x} w_{m-\frac{3}{2}}-3 \delta_{x} w_{m-\frac{1}{2}}\right) w_{m-1} \\
= & h \sum_{i=1}^{m-2}\left(\delta_{x}^{2} v_{i}\right) w_{i}+\frac{1}{h}\left(v_{m-2}-3 v_{m-1}\right) w_{m-1} \\
= & \sum_{i=0}^{m-2}\left(\delta_{x} v_{i+\frac{1}{2}}\right)\left(w_{i}-w_{i+1}\right)+\left(\delta_{x} v_{m-\frac{3}{2}}\right) w_{m-1}+\frac{1}{h}\left(v_{m-2}-3 v_{m-1}\right) w_{m-1} \\
= & -h \sum_{i=0}^{m-2}\left(\delta_{x} v_{i+\frac{1}{2}}\right) v_{i}+\frac{1}{h}\left(v_{m-1}-v_{m-2}+v_{m-2}-3 v_{m-1}\right) w_{m-1} \\
= & \frac{1}{2} \sum_{i=0}^{m-2}\left(v_{i+1}-v_{i}\right)^{2}+\frac{1}{2} v_{0}^{2}-\frac{1}{2} v_{m-1}^{2}-2 v_{m-1} \frac{w_{m-1}-w_{m}}{h} \\
= & \frac{1}{2} \sum_{i=1}^{m-1}\left(v_{i}-v_{i-1}\right)^{2}+\frac{1}{2} v_{0}^{2}-\frac{1}{2} v_{m-1}^{2}+2 v_{m-1}^{2} \\
= & \frac{1}{2} \sum_{i=1}^{m-1}\left(v_{i}-v_{i-1}\right)^{2}+\frac{1}{2} v_{0}^{2}+\frac{3}{2} v_{m-1}^{2} \\
= & \frac{1}{2} h^{2} \sum_{i=1}^{m-1}\left(\delta_{x}^{2} w_{i}\right)^{2}+\frac{1}{2}\left(\delta_{x} w_{\frac{1}{2}}\right)^{2}+\frac{3}{2}\left(\delta_{x} w_{m-\frac{1}{2}}\right)^{2}
\end{aligned}
$$

This completes the proof.

Lemma 2.4. Suppose $g(x) \in C^{4}(c-h, c+2 h)$. Then we have

$$
g^{\prime \prime \prime}(c)-\frac{1}{h^{3}}[g(c+2 h)-3 g(c+h)+3 g(c)-g(c-h)]=\mathcal{O}(h) .
$$

Lemma 2.5. Suppose $g(x) \in C^{2}(c-h, c)$. Then there exists at least $a \xi \in(c-2 h, c)$ such that

$$
g^{\prime}(c)-\frac{1}{h}[g(c)-g(c-h)]=\frac{h}{2} g^{\prime \prime}(\xi) .
$$

Lemma 2.6. Suppose $g(x) \in C^{3}(c-2 h, c)$. Then there exists at least an $\eta \in(c-2 h, c)$ such that

$$
g^{\prime}(c)-\left\{2 \frac{1}{h}[g(c)-g(c-h)]-\frac{1}{2 h}[g(c)-g(c-2 h)]\right\}=\frac{h^{2}}{3} g^{\prime \prime \prime}(\eta),
$$


or

$$
g^{\prime}(c)-\left\{\frac{3}{2} \cdot \frac{1}{h}[g(c)-g(c-h)]-\frac{1}{2} \cdot \frac{1}{h}[g(c-h)-g(c-2 h)]\right\}=\frac{h^{2}}{3} g^{\prime \prime \prime}(\eta) .
$$

If $g(x) \in C^{4}(c-2 h, c)$. Then we have

$$
\begin{aligned}
& g^{\prime}(c)-\left\{\frac{3}{2} \cdot \frac{1}{h}[g(c)-g(c-h)]-\frac{1}{2} \cdot \frac{1}{h}[g(c-h)-g(c-2 h)]\right\} \\
= & \frac{h^{2}}{3} g^{\prime \prime \prime}(c)+\mathcal{O}\left(h^{3}\right) .
\end{aligned}
$$

To end this section, we recall that the solution of problem (1.1a)-(1.1c) has the following conservation law.

Lemma 2.7. Let $u(x, t)$ be the solution of problem (1.1a)-(1.1c). Denote

$$
E(t)=\int_{0}^{L} u^{2}(x, t) d x+\int_{0}^{t} u_{x}^{2}(0, s) d s,
$$

then we have

$$
E(t)=E(0), \quad 0<t \leq T .
$$

We call (2.4) the conservation of energy. By identity (2.4), we obviously know

$$
\int_{0}^{L} u^{2}(x, t) d x \leq \int_{0}^{L} u^{2}(x, 0) d x, \quad 0 \leq t \leq T .
$$

\section{A two-level nonlinear finite difference scheme}

In this section, we construct a two-level finite difference scheme and discuss its existence, conservation, boundedness and convergence. We suppose that the problem (1.1a)-(1.1c) has a solution $u(x, t) \in C^{3}\left(0, T ; C^{4}(0, L)\right)$.

\subsection{The derivation of the finite difference scheme}

Define the grid function

$$
U_{i}^{k}=u\left(x_{i}, t_{k}\right), \quad 0 \leq i \leq m, \quad 0 \leq k \leq n .
$$

Considering Eq. (1.1a) at the points $\left(x_{i}, t_{k+\frac{1}{2}}\right)$, we have

$$
\begin{aligned}
& u_{t}\left(x_{i}, t_{k+\frac{1}{2}}\right)+\gamma u\left(x_{i}, t_{k+\frac{1}{2}}\right) u_{x}\left(x_{i}, t_{k+\frac{1}{2}}\right)+u_{x x x}\left(x_{i}, t_{k+\frac{1}{2}}\right) \\
= & 0, \quad 1 \leq i \leq m-2, \quad 0 \leq k \leq n-1 .
\end{aligned}
$$


Using Lemma 2.4, we have

$$
\begin{gathered}
\delta_{t} U_{i}^{k+\frac{1}{2}}+\gamma \psi\left(U^{k+\frac{1}{2}}, U^{k+\frac{1}{2}}\right)_{i}+\delta_{x}^{2}\left(\delta_{x} U_{i+\frac{1}{2}}^{k+\frac{1}{2}}\right)=P_{i}^{k+\frac{1}{2}}, \\
1 \leq i \leq m-2, \quad 0 \leq k \leq n-1
\end{gathered}
$$

and there is a constant $c_{0}>0$ such that

$$
\left|P_{i}^{k+\frac{1}{2}}\right| \leq c_{0}\left(h+\tau^{2}\right), \quad 1 \leq i \leq m-2, \quad 0 \leq k \leq n-1 .
$$

Now we consider the approximation of the boundary condition

$$
u_{x}(L, t)=0, \quad 0 \leq t \leq T
$$

Using Lemma 2.5, we have

$$
u_{x}\left(x_{m}, t_{k}\right)=\frac{1}{h}\left(U_{m}^{k}-U_{M-1}^{k}\right)+\frac{h}{2} u_{x x}\left(\xi_{m}^{k}, t_{k}\right), \quad \xi_{m}^{k} \in\left(x_{m-1}, x_{m}\right), \quad 1 \leq k \leq n .
$$

Omitting the small term, we easily obtain a one order approximation

$$
\frac{1}{h}\left(U_{m}^{k}-U_{m-1}^{k}\right) \approx 0, \quad 1 \leq k \leq n
$$

Our aim is to get a third order approximation for (3.5). From (1.1a) and (1.1c), we know

$$
u_{x x x}\left(x_{m}, t\right)=0, \quad 0 \leq t \leq T .
$$

Using Lemma 2.6, (1.1a) and (3.6), we get

$$
\begin{aligned}
& u_{x}\left(x_{m}, t\right)=\left\{\frac{3}{2} \cdot \frac{1}{h}\left[u\left(x_{m}, t\right)-u\left(x_{m}-h, t\right)\right]-\frac{1}{2} \cdot \frac{1}{h}\left[u\left(x_{m}-h, t\right)-u\left(x_{m}-2 h, t\right)\right]\right\} \\
& +\frac{h^{2}}{3} u_{x x x}\left(x_{m}, t\right)+\mathcal{O}\left(h^{3}\right) \\
& =\left\{\frac{3}{2} \cdot \frac{1}{h}\left[u\left(x_{m}, t\right)-u\left(x_{m}-h, t\right)\right]-\frac{1}{2} \cdot \frac{1}{h}\left[u\left(x_{m}-h, t\right)-u\left(x_{m}-2 h, t\right)\right]\right\} \\
& +\frac{h^{2}}{2} u_{x x x}\left(x_{m}, t\right)+\mathcal{O}\left(h^{3}\right) \\
& =\left\{\frac{3}{2} \cdot \frac{1}{h}\left[u\left(x_{m}, t\right)-u\left(x_{m}-h, t\right)\right]-\frac{1}{2} \cdot \frac{1}{h}\left[u\left(x_{m}-h, t\right)-u\left(x_{m}-2 h, t\right)\right]\right\} \\
& +\frac{h^{2}}{2} u_{x x x}\left(x_{m}-h, t\right)+\mathcal{O}\left(h^{3}\right) \\
& =\left\{\frac{3}{2} \cdot \frac{1}{h}\left[u\left(x_{m}, t\right)-u\left(x_{m}-h, t\right)\right]-\frac{1}{2} \cdot \frac{1}{h}\left[u\left(x_{m}-h, t\right)-u\left(x_{m}-2 h, t\right)\right]\right\} \\
& -\frac{h^{2}}{2}\left[u_{t}\left(x_{m}-h, t\right)+\gamma\left(u u_{x}\right)\left(x_{m}-h, t\right)\right]+\mathcal{O}\left(h^{3}\right) .
\end{aligned}
$$


Letting $t=t_{k}$ and $t=t_{k+1}$ in the above equality and averaging the results, we obtain

$$
\begin{aligned}
& \frac{1}{2}\left(u_{x}\left(x_{m}, t_{k}\right)+u_{x}\left(x_{m}, t_{k+1}\right)\right) \\
= & {\left[\frac{3}{2} \cdot \delta_{x} U_{m-\frac{1}{2}}^{k+\frac{1}{2}}-\frac{1}{2} \cdot \delta_{x} U_{m-\frac{3}{2}}^{k+\frac{1}{2}}\right]-\frac{h^{2}}{2}\left[\delta_{t} U_{m-1}^{k+\frac{1}{2}}+\gamma \psi\left(U^{k+\frac{1}{2}}, U^{k+\frac{1}{2}}\right)_{m-1}\right] } \\
& \quad+\mathcal{O}\left(h^{3}\right)+\mathcal{O}\left(h^{2}\left(h^{2}+\tau^{2}\right)\right)=0, \quad 0 \leq k \leq n-1 .
\end{aligned}
$$

That means that

$$
\left[\frac{3}{2} \cdot \delta_{x} U_{m-\frac{1}{2}}^{k+\frac{1}{2}}-\frac{1}{2} \cdot \delta_{x} U_{m-\frac{3}{2}}^{k+\frac{1}{2}}\right]-\frac{h^{2}}{2}\left[\delta_{t} U_{m-1}^{k+\frac{1}{2}}+\gamma \psi\left(U^{k+\frac{1}{2}}, U^{k+\frac{1}{2}}\right)_{m-1}\right] \approx 0
$$

is a third order approximation of

$$
\frac{1}{2}\left(u_{x}\left(x_{m}, t_{k}\right)+u_{x}\left(x_{m}, t_{k+1}\right)\right)=0 .
$$

Multiplying (3.7) by $\left(-\frac{2}{h^{2}}\right)$, we have

$$
\begin{aligned}
& \delta_{t} U_{m-1}^{k+\frac{1}{2}}+\gamma \psi\left(U^{k+\frac{1}{2}}, U^{k+\frac{1}{2}}\right)_{m-1}+\frac{1}{h^{2}}\left(\delta_{x} U_{m-\frac{3}{2}}^{k+\frac{1}{2}}-3 \delta_{x} U_{m-\frac{1}{2}}^{k+\frac{1}{2}}\right) \\
= & P_{m-1}^{k+\frac{1}{2}}, \quad 0 \leq k \leq n-1,
\end{aligned}
$$

and there is a constant $c_{1}>0$ such that

$$
\left|P_{m-1}^{k+\frac{1}{2}}\right| \leq c_{1}\left(h+\tau^{2}\right), \quad 0 \leq k \leq n-1 .
$$

Noticing the initial-boundary value conditions (1.1b)-(1.1c), we have

$$
\begin{aligned}
& U_{i}^{0}=\varphi\left(x_{i}\right), \quad 1 \leq i \leq m-1, \\
& U_{0}^{k}=0, \quad U_{m}^{k}=0, \quad 0 \leq k \leq n .
\end{aligned}
$$

Omitting the small terms in (3.3) and (3.8), denoting by $u_{i}^{k}$ the numerical approximation of $U_{i}^{k}$, we obtain a two-level nonlinear difference scheme

$$
\begin{gathered}
\delta_{t} u_{i}^{k+\frac{1}{2}}+\gamma \psi\left(u^{k+\frac{1}{2}}, u^{k+\frac{1}{2}}\right)_{i}+\delta_{x}^{2}\left(\delta_{x} u_{i+\frac{1}{2}}^{k+\frac{1}{2}}\right)=0, \\
1 \leq i \leq m-2, \quad 0 \leq k \leq n-1, \\
\delta_{t} u_{m-1}^{k+\frac{1}{2}}+\gamma \psi\left(u^{k+\frac{1}{2}}, u^{k+\frac{1}{2}}\right)_{m-1}+\frac{1}{h^{2}}\left(\delta_{x} u_{m-\frac{3}{2}}^{k+\frac{1}{2}}-3 \delta_{x} u_{m-\frac{1}{2}}^{k+\frac{1}{2}}\right)=0, \\
0 \leq k \leq n-1, \\
1 \leq i \leq m-1, \\
u_{i}^{0}=\varphi\left(x_{i}\right), \quad 0 \leq k \leq n . \\
u_{0}^{k}=0, \quad u_{m}^{k}=0, \quad 0
\end{gathered}
$$


Denote

$$
v_{m}^{k+\frac{1}{2}}=0, \quad v_{i}^{k+\frac{1}{2}}=\delta_{x} u_{i+\frac{1}{2}}^{k+\frac{1}{2}}, \quad i=m-1, m-2, \cdots, 0, \quad 0 \leq k \leq n-1 .
$$

Rewriting (3.11a)-(3.11d), we obtain

$$
\begin{array}{ll}
\delta_{t} u_{i}^{k+\frac{1}{2}}+\gamma \psi\left(u^{k+\frac{1}{2}}, u^{k+\frac{1}{2}}\right)_{i}+\delta_{x}^{2} v_{i}^{k+\frac{1}{2}}=0, \quad 1 \leq i \leq m-2, & 0 \leq k \leq n-1, \\
\delta_{t} u_{m-1}^{k+\frac{1}{2}}+\gamma \psi\left(u^{k+\frac{1}{2}}, u^{k+\frac{1}{2}}\right)_{m-1}+\frac{1}{h^{2}}\left(v_{m-2}^{k+\frac{1}{2}}-3 v_{m-1}^{k+\frac{1}{2}}\right)=0, & 0 \leq k \leq n-1, \\
u_{i}^{0}=\varphi\left(x_{i}\right), & 1 \leq i \leq m-1, \\
u_{0}^{k}=0, \quad u_{m}^{k}=0, \quad 0 \leq k \leq n, & \\
v_{m}^{k+\frac{1}{2}}=0, \quad v_{i}^{k+\frac{1}{2}}=\delta_{x} u_{i+\frac{1}{2}}^{k+\frac{1}{2}}, \quad i=m-1, m-2, \cdots, 0, \quad 0 \leq k \leq n-1 .
\end{array}
$$

Remark 3.1. Noticing that

$$
\begin{aligned}
\frac{2}{3} u_{x x x}\left(x_{m-1}, t\right)=\frac{1}{h^{2}} & {\left[\frac{1}{h}\left(u\left(x_{m}-h, t\right)-u\left(x_{m}-2 h, t\right)\right)\right.} \\
& \left.-3 \cdot \frac{1}{h}\left(u\left(x_{m}, t\right)-u\left(x_{m}-h, t\right)\right)+2 u_{x}\left(x_{m}, t\right)\right]+\mathcal{O}(h),
\end{aligned}
$$

we can regard (3.8) as a disretization of the equality

$$
\begin{aligned}
& u_{t}\left(x_{m-1}, t_{k+\frac{1}{2}}\right)+\gamma u\left(x_{m-1}, t_{k+\frac{1}{2}}\right) u_{x}\left(x_{m-1}, t_{k+\frac{1}{2}}\right)+\frac{2}{3} u_{x x x}\left(x_{m-1}, t_{k+\frac{1}{2}}\right) \\
= & \mathcal{O}(h), \quad 0 \leq k \leq n-1,
\end{aligned}
$$

with the truncation of an order of $\mathcal{O}\left(h+\tau^{2}\right)$.

Remark 3.2. Suppose that $g(x) \in C^{(5)}\left[x_{m-1}, x_{m+1}\right]$, where $x_{m-1}=x_{m}-h, x_{m+1}=$ $x_{m}+h$. Applying the Taylor expansion, we have

$$
\frac{1}{2 h}\left[g\left(x_{m+1}\right)-g\left(x_{m-1}\right)\right]=g^{\prime}\left(x_{m}\right)+\frac{h^{2}}{6} g^{\prime \prime \prime}\left(x_{m}\right)+\frac{h^{4}}{120} g^{(5)}(\xi), \quad \xi \in\left(x_{m-1}, x_{m+1}\right) .
$$

If the problem (1.1a)-(1.1c) can be extended to be defined in the domain $[0, L+h] \times$ $[0, T]$ and also has a smooth solution, then

$$
\begin{aligned}
\frac{1}{2 h}\left(U_{m+1}^{k}-U_{m-1}^{k}\right) & =u_{x}\left(x_{m}, t_{k}\right)+\frac{h^{2}}{6} u_{x x x}\left(x_{m}, t_{k}\right)+\frac{h^{4}}{120} u_{x x x x x}\left(\xi_{k}, t_{k}\right) \\
& =\frac{h^{4}}{120} u_{x x x x x}\left(\xi_{k}, t_{k}\right)=\mathcal{O}\left(h^{4}\right), \quad \xi_{k} \in\left(x_{m-1}, x_{m+1}\right),
\end{aligned}
$$

which follows that

$$
\frac{1}{2 h}\left(U_{m+1}^{k+\frac{1}{2}}-U_{m-1}^{k+\frac{1}{2}}\right)=\mathcal{O}\left(h^{4}\right), \quad 0 \leq k \leq n-1 .
$$

In addition, (3.3) is also valid for $i=m-1$. Using these two equalities to remove the fictitious values $\left\{U_{m+1}^{k}\right\}$, we can obtain (3.8) and (3.9) straightforwardly. 


\subsection{Existence, boundedness and uniqueness of the numerical solution}

First, we recall the Browder fixed-point theorem.

Theorem $3.1([1,6])$. Suppose $(H,(\cdot, \cdot))$ is a finite dimensional inner space, $\|\cdot\|$ is the induced norm, $\Pi: H \rightarrow H$ is a continuous operator on $(H,(\cdot, \cdot))$. If there exists a constant $\alpha>0, \forall z \in H,\|z\|=\alpha,(\Pi(z), z) \geq 0$, then there exists a $z^{*} \in H,\left\|z^{*}\right\| \leq \alpha$ such that $\Pi\left(z^{*}\right)=0$.

Theorem 3.2. There is a solution of the finite difference scheme (3.11a)-(3.11d).

Proof. According to (3.11c)-(3.11d), we get $u^{0}$. Suppose that the solution $u^{k}$ at the $k$-th time level is known. Let

$$
w_{i}=\left(u_{i}^{k+1}+u_{i}^{k}\right) / 2, \quad 0 \leq i \leq m .
$$

It can be seen from finite difference scheme (3.11a)-(3.11b) and (3.11d) that

$$
\begin{aligned}
& \frac{2}{\tau}\left(w_{i}-u_{i}^{k}\right)+\gamma \psi(w, w)_{i}+\delta_{x}^{2}\left(\delta_{x} w_{i+\frac{1}{2}}\right)=0, \quad 1 \leq i \leq m-2, \\
& \frac{2}{\tau}\left(w_{m-1}-u_{m-1}^{k}\right)+\gamma \psi(w, w)_{m-1}+\frac{1}{h^{2}}\left(\delta_{x} w_{m-\frac{3}{2}}-3 \delta_{x} w_{m-\frac{1}{2}}\right)=0, \\
& w_{0}=0, \quad w_{m}=0 .
\end{aligned}
$$

If we have determined $w$, then

$$
u_{i}^{k+1}=2 w_{i}-u_{i}^{k}, \quad 0 \leq i \leq m .
$$

For $w \in \stackrel{\mathscr{U}}{h}_{h}$, define the operator

$$
\Pi(w)_{i}= \begin{cases}\frac{2}{\tau}\left(w_{i}-u_{i}^{k}\right)+\gamma \psi(w, w)_{i}+\delta_{x}^{2}\left(\delta_{x} w_{i+\frac{1}{2}}\right), & 1 \leq i \leq m-2, \\ \frac{2}{\tau}\left(w_{m-1}-u_{m-1}^{k}\right)+\gamma \psi(w, w)_{m-1} & \\ +\frac{1}{h^{2}}\left(\delta_{x} w_{m-\frac{3}{2}}-3 \delta_{x} w_{m-\frac{1}{2}}\right), & i=m-1 .\end{cases}
$$

It is easy to get

$$
\begin{gathered}
(\Pi(w), w)=\frac{2}{\tau}\left[\|w\|^{2}-\left(u^{k}, w\right)\right]+\gamma(\psi(w, w), w)+h \sum_{i=1}^{m-2}\left(\delta_{x}^{2}\left(\delta_{x} w_{i+\frac{1}{2}}\right)\right) w_{i} \\
+\frac{1}{h}\left(\delta_{x} w_{m-\frac{3}{2}}-3 \delta_{x} w_{m-\frac{1}{2}}\right) w_{m-1}
\end{gathered}
$$

By Lemma 2.3 and Lemma 2.1, we have

$$
(\Pi(w), w) \geq \frac{2}{\tau}\left[\|w\|^{2}-\left(u^{k}, w\right)\right] \geq \frac{2}{\tau}\|w\|\left(\|w\|-\left\|u^{k}\right\|\right) .
$$


When $\|w\|=\left\|u^{k}\right\|,(\Pi(w), w) \geq 0$. By Browder Theorem 3.1, there is a $w^{*} \in \dot{\mathcal{U}}_{h}$ and $\left\|w^{*}\right\| \leq\left\|u^{k}\right\|$ satisfying

$$
\Pi\left(w^{*}\right)=0 .
$$

The proof of this theorem is finished.

Theorem 3.3. Suppose $\left\{u_{i}^{k} \mid 0 \leq i \leq m, 0 \leq k \leq n\right\}$ is the solution of finite difference scheme (3.11a)-(3.11d). Denote

$$
E^{k}=\left\|u^{k}\right\|^{2}+\tau \sum_{l=0}^{k-1}\left[\left(\delta_{x} u_{\frac{1}{2}}^{l+\frac{1}{2}}\right)^{2}+3\left(\delta_{x} u_{m-\frac{1}{2}}^{l+\frac{1}{2}}\right)^{2}+h^{2} \sum_{i=1}^{m-1}\left(\delta_{x}^{2} u_{i}^{l+\frac{1}{2}}\right)^{2}\right],
$$

we have

$$
E^{k}=E^{0}, \quad 1 \leq k \leq n
$$

Proof. Multiplying (3.11a) and (3.11b) by $h u_{i}^{k+\frac{1}{2}}$ and $h u_{m-1}^{k+\frac{1}{2}}$ respectively, then summing up the results, we obtain

$$
\begin{aligned}
& \left(\delta_{t} u^{k+\frac{1}{2}}, u^{k+\frac{1}{2}}\right)+\gamma\left(\psi\left(u^{k+\frac{1}{2}}, u^{k+\frac{1}{2}}\right), u^{k+\frac{1}{2}}\right) \\
& \quad+h \sum_{i=1}^{m-2}\left(\delta_{x}^{2} \delta_{x} u_{i+\frac{1}{2}}^{k+\frac{1}{2}}\right) u_{i}^{k+\frac{1}{2}}+\frac{1}{h}\left(\delta_{x} u_{m-\frac{3}{2}}^{k+\frac{1}{2}}-3 \delta_{x} u_{m-\frac{1}{2}}^{k+\frac{1}{2}}\right) u_{m-1}^{k+\frac{1}{2}}=0
\end{aligned}
$$

Using Lemma 2.1 and Lemma 2.3, we get

$$
\begin{aligned}
& \frac{1}{2 \tau}\left(\left\|u^{k+1}\right\|^{2}-\left\|u^{k}\right\|^{2}\right)+\frac{1}{2} h^{2} \sum_{i=1}^{m-1}\left(\delta_{x}^{2} u_{i}^{k+\frac{1}{2}}\right)^{2}+\frac{1}{2}\left(\delta_{x} u_{\frac{1}{2}}^{k+\frac{1}{2}}\right)^{2}+\frac{3}{2}\left(\delta_{x} u_{m-\frac{1}{2}}^{k+\frac{1}{2}}\right)^{2} \\
= & 0, \quad 0 \leq k \leq n-1 .
\end{aligned}
$$

Replacing $k$ by $l$ and summing $l$ from 0 to $k$, we get

$$
\begin{aligned}
& \left\|u^{k+1}\right\|^{2}+\tau \sum_{l=0}^{k}\left[\left(\delta_{x} u_{\frac{1}{2}}^{l+\frac{1}{2}}\right)^{2}+3\left(\delta_{x} u_{m-\frac{1}{2}}^{l+\frac{1}{2}}\right)^{2}+h^{2} \sum_{i=1}^{m-1}\left(\delta_{x}^{2} u_{i}^{l+\frac{1}{2}}\right)^{2}\right] \\
= & \left\|u^{0}\right\|^{2}, \quad 0 \leq k \leq n-1,
\end{aligned}
$$

that is

$$
E^{k+1}=E^{0}, \quad 0 \leq k \leq n-1 .
$$

This completes the proof of the theorem.

\section{Noticing}

$$
u_{x}(L, t)=0, \quad 0 \leq t \leq T,
$$


the $E(t)$ in Lemma 2.7 can be written as

$$
E(t)=\int_{0}^{L} u^{2}(x, t) d x+\int_{0}^{t}\left[u_{x}^{2}(0, s)+3 u_{x}^{2}(L, t)\right] d s .
$$

The $E^{k}$ in Theorem 3.3 can be written as

$$
E^{k}=\left\|u^{k}\right\|^{2}+\tau \sum_{l=0}^{k-1}\left[\left(\delta_{x} u_{\frac{1}{2}}^{l+\frac{1}{2}}\right)^{2}+3\left(\delta_{x} u_{m-\frac{1}{2}}^{l+\frac{1}{2}}\right)^{2}\right]+h \cdot \tau \sum_{l=0}^{k-1}\left[h \sum_{i=1}^{m-1}\left(\delta_{x}^{2} u_{i}^{l+\frac{1}{2}}\right)^{2}\right] .
$$

In addition, the last term in the above expression is a small quantity with order of $\mathcal{O}(h)$. It is obvious that $E^{k}$ is a discrete version of $E\left(t_{k}\right)$. It follows from Theorem 3.3 that $\|w\| \leq c$.

Theorem 3.4. Denote $c=\left\|u^{0}\right\|$. If $c|\gamma| \sqrt{L} \frac{\tau}{h^{2}}<1$, then the solution of finite difference scheme (3.14a)-(3.14c) is unique.

Proof. Observing the proof of Theorem 3.2, it can be seen that we only need to prove the solution of difference scheme (3.14a)-(3.14c) is unique. Suppose that the difference scheme (3.14a)-(3.14c) has an another solution $\left\{v_{i} \mid 0 \leq i \leq m\right\}$, namely, $\left\{v_{i} \mid 0 \leq i \leq m\right\}$ satisfies

$$
\begin{aligned}
& \frac{2}{\tau}\left(v_{i}-u_{i}^{k}\right)+\gamma \psi(v, v)_{i}+\delta_{x}^{2}\left(\delta_{x} v_{i+\frac{1}{2}}\right)=0, \quad 1 \leq i \leq m-2, \\
& \frac{2}{\tau}\left(v_{m-1}-u_{m-1}^{k}\right)+\gamma \psi(v, v)_{m-1}+\frac{1}{h^{2}}\left(\delta_{x} v_{m-\frac{3}{2}}-3 \delta_{x} v_{m-\frac{1}{2}}\right)=0 \\
& v_{0}=0, \quad v_{m}=0 .
\end{aligned}
$$

Denote

$$
z_{i}=w_{i}-v_{i}, \quad 0 \leq i \leq m .
$$

Subtracting (3.22a)-(3.22c) from (3.14a)-(3.14c), it follows

$$
\begin{aligned}
& \frac{2}{\tau} z_{i}+\gamma\left[\psi(w, w)_{i}-\psi(v, v)_{i}\right]+\delta_{x}^{2}\left(\delta_{x} z_{i+\frac{1}{2}}\right)=0, \quad 1 \leq i \leq m-2, \\
& \frac{2}{\tau} z_{m-1}+\gamma\left[\psi(w, w)_{m-1}-\psi(v, v)_{m-1}\right]+\frac{1}{h^{2}}\left(\delta_{x} z_{m-\frac{3}{2}}-3 \delta_{x} z_{m-\frac{1}{2}}\right)=0, \\
& z_{0}=0, \quad z_{m}=0 .
\end{aligned}
$$

Multiplying (3.23a) by $h z_{i}$ and (3.23b) and $h z_{m-1}$, respectively, then summing up the results, we get

$$
\begin{gathered}
\frac{2}{\tau}\|z\|^{2}+\gamma(\psi(w, w)-\psi(v, v), z)+h \sum_{i=1}^{m-2}\left(\delta_{x}^{2} \delta_{x} z_{i+\frac{1}{2}}\right) z_{i} \\
+\frac{1}{h}\left(-3 \delta_{x} z_{m-\frac{1}{2}}+\delta_{x} z_{m-\frac{3}{2}}\right) z_{m-1}=0
\end{gathered}
$$


Notice

$$
\begin{aligned}
& \psi(w, w)_{i}-\psi(v, v)_{i} \\
= & \psi(w, w)_{i}-\psi(w-z, w-z)_{i} \\
= & \psi(z, w)_{i}+\psi(w, z)_{i}-\psi(z, z)_{i} .
\end{aligned}
$$

Using Lemma 2.1, we have

$$
(\psi(w, w)-\psi(v, v), z)=(\psi(z, w)+\psi(w, z)-\psi(z, z), z)=(\psi(z, w), z) .
$$

Using Lemma 2.3, we have

$$
h \sum_{i=1}^{m-2}\left(\delta_{x}^{2} \delta_{x} z_{i+\frac{1}{2}}\right) z_{i}+\frac{1}{h}\left(-3 \delta_{x} z_{m-\frac{1}{2}}+\delta_{x} z_{m-\frac{3}{2}}\right) z_{m-1} \geq 0 .
$$

Substituting (3.26) and (3.27) into (3.24) and noticing Lemma 2.2, we obtain

$$
\begin{aligned}
\frac{2}{\tau}\|z\|^{2} & \leq-\gamma(\psi(z, w), z)=-\frac{\gamma h}{3} \sum_{i=1}^{m-1}\left[z_{i} \Delta_{x} w_{i}+\Delta_{x}(z w)_{i}\right] z_{i} \\
& =\frac{\gamma h}{3} \sum_{i=1}^{m-1}\left[w_{i} \Delta_{x}\left(z_{i}^{2}\right)+(z w)_{i} \Delta z_{i}\right] \leq \frac{|\gamma|}{3}\left(2\|z\|_{\infty}\|w\| \cdot|z|_{1}+\|z\|_{\infty}\|w\| \cdot|z|_{1}\right) \\
& =|\gamma|\|w\| \cdot\|z\|_{\infty} \cdot|z|_{1} \leq c|\gamma| \frac{\sqrt{L}}{2}|z|_{1}^{2} \leq c|\gamma| \frac{2 \sqrt{L}}{h^{2}}\|z\|^{2} .
\end{aligned}
$$

When $c|\gamma| \sqrt{L} \frac{\tau}{h^{2}}<1$, we have $\|z\|=0$. The proof of this theorem is completed.

\subsection{Convergence}

Next, we strictly prove the convergence of the finite difference scheme.

Theorem 3.5. Suppose $u(x, t) \in C^{3}\left(0, T ; C^{4}(0, L)\right)$ be the solution of (1.1a)-(1.1c) and $\left\{u_{i}^{k} \mid 0 \leq i \leq m, 0 \leq k \leq n\right\}$ be the solution of (3.11a)-(3.11d). Denote

$$
\begin{aligned}
e_{i}^{k} & =U_{i}^{k}-u_{i}^{k}, \quad 0 \leq i \leq m, \quad 0 \leq k \leq n, \\
F^{k} & =\sqrt{\left\|e^{k}\right\|^{2}+\tau \sum_{l=0}^{k-1}\left[\left(\delta_{x} e_{\frac{1}{2}}^{l+\frac{1}{2}}\right)^{2}+3\left(\delta_{x} e_{m-\frac{1}{2}}^{l+\frac{1}{2}}\right)^{2}+h^{2} \sum_{i=1}^{m-1}\left(\delta_{x}^{2} e_{i}^{k+\frac{1}{2}}\right)^{2}\right]}, \quad 0 \leq k \leq n .
\end{aligned}
$$

Then there exists a constant $c_{2}>0$ such that

$$
F^{k} \leq c_{2}\left(h+\tau^{2}\right), \quad 0 \leq k \leq n .
$$


Proof. Subtracting (3.11a)-(3.11d) from (3.3), (3.8), (3.10a)-(3.10b), we obtain the error equations

$$
\begin{aligned}
& \delta_{t} e_{i}^{k+\frac{1}{2}}+\gamma\left[\psi\left(U^{k+\frac{1}{2}}, U^{k+\frac{1}{2}}\right)_{i}-\psi\left(u^{k+\frac{1}{2}}, u^{k+\frac{1}{2}}\right)_{i}\right]+\delta_{x}^{2} \delta_{x} e_{i+\frac{1}{2}}^{k+\frac{1}{2}} \\
& =P_{i}^{k+\frac{1}{2}}, \quad 1 \leq i \leq m-2, \quad 0 \leq k \leq n-1, \\
& \delta_{t} e_{m-1}^{k+\frac{1}{2}}+\gamma\left[\psi\left(U^{k+\frac{1}{2}}, U^{k+\frac{1}{2}}\right)_{m-1}-\psi\left(u^{k+\frac{1}{2}}, u^{k+\frac{1}{2}}\right)_{m-1}\right]+\frac{1}{h^{2}}\left(\delta_{x} e_{m-\frac{3}{2}}^{k+\frac{1}{2}}-3 \delta_{x} e_{m-\frac{1}{2}}^{k+\frac{1}{2}}\right) \\
& =P_{m-1}^{k+\frac{1}{2}}, \quad 0 \leq k \leq n-1, \\
& e_{i}^{0}=0, \quad 1 \leq i \leq m-1, \\
& e^{k}=0, \quad e_{m}^{k}=0, \quad 0 \leq k \leq n
\end{aligned}
$$

Multiplying (3.29a) and (3.29b) by $h e_{i}^{k+\frac{1}{2}}$ and $h e_{m-1}^{k+\frac{1}{2}}$, respectively and summing up the results, we get

$$
\begin{gathered}
\left(\delta_{t} e^{k+\frac{1}{2}}, e^{k+\frac{1}{2}}\right)+\gamma\left(\psi\left(U^{k+\frac{1}{2}}, U^{k+\frac{1}{2}}\right)-\psi\left(u^{k+\frac{1}{2}}, u^{k+\frac{1}{2}}\right), e^{k+\frac{1}{2}}\right)+h \sum_{i=1}^{m-2}\left(\delta_{x}^{2} \delta_{x} e_{i+\frac{1}{2}}^{k+\frac{1}{2}}\right) e_{i}^{k+\frac{1}{2}} \\
+\frac{1}{h}\left(-3 \delta_{x} e_{m-\frac{1}{2}}^{k+\frac{1}{2}}+\delta_{x} e_{m-\frac{3}{2}}^{k+\frac{1}{2}}\right) e_{m-1}^{k+\frac{1}{2}}=\left(P^{k+\frac{1}{2}}, e^{k+\frac{1}{2}}\right), \quad 0 \leq k \leq n-1 .
\end{gathered}
$$

For the second term on the left hand of (3.30), using Lemma 2.1, we have

$$
\begin{aligned}
& \left(\psi\left(U^{k+\frac{1}{2}}, U^{k+\frac{1}{2}}\right)-\psi\left(u^{k+\frac{1}{2}}, u^{k+\frac{1}{2}}\right), e^{k+\frac{1}{2}}\right) \\
= & \left(\psi\left(U^{k+\frac{1}{2}}, U^{k+\frac{1}{2}}\right)-\psi\left(U^{k+\frac{1}{2}}-e^{k+\frac{1}{2}}, U^{k+\frac{1}{2}}-e^{k+\frac{1}{2}}\right), e^{k+\frac{1}{2}}\right) \\
= & \left(\psi\left(e^{k+\frac{1}{2}}, U^{k+\frac{1}{2}}\right)+\psi\left(U^{k+\frac{1}{2}}, e^{k+\frac{1}{2}}\right)-\psi\left(e^{k+\frac{1}{2}}, e^{k+\frac{1}{2}}\right), e^{k+\frac{1}{2}}\right) \\
= & \left(\psi\left(e^{k+\frac{1}{2}}, U^{k+\frac{1}{2}}\right), e^{k+\frac{1}{2}}\right) \\
= & \frac{1}{3} h \sum_{i=1}^{m-1}\left[e_{i}^{k+\frac{1}{2}} \Delta_{x} U_{i}^{k+\frac{1}{2}}+\Delta_{x}(e U)_{i}^{k+\frac{1}{2}}\right] e_{i}^{k+\frac{1}{2}} \\
= & \frac{1}{3} h \sum_{i=1}^{m-1}\left(\Delta_{x} U_{i}^{k+\frac{1}{2}}\right)\left(e_{i}^{k+\frac{1}{2}}\right)^{2}+\frac{1}{6} \sum_{i=1}^{m-1}\left(e_{i+1}^{k+\frac{1}{2}} U_{i+1}^{k+\frac{1}{2}}-e_{i-1}^{k+\frac{1}{2}} U_{i-1}^{k+\frac{1}{2}}\right) e_{i}^{k+\frac{1}{2}} \\
= & \frac{1}{3} h \sum_{i=1}^{m-1}\left(\Delta_{x} U_{i}^{k+\frac{1}{2}}\right)\left(e_{i}^{k+\frac{1}{2}}\right)^{2}+\frac{1}{6} h \sum_{i=1}^{m-1} e_{i+1}^{k+\frac{1}{2}} e_{i}^{k+\frac{1}{2}} \delta_{x} U_{i+\frac{1}{2}}^{k+\frac{1}{2}} .
\end{aligned}
$$

Denote

$$
c_{3}=\max _{0 \leq x \leq L, 0 \leq t \leq T}\left|u_{x}(x, t)\right|
$$


Then we have

$$
\begin{gathered}
-\left(\psi\left(U^{k+\frac{1}{2}}, U^{k+\frac{1}{2}}\right)-\psi\left(u^{k+\frac{1}{2}}, u^{k+\frac{1}{2}}\right), e^{k+\frac{1}{2}}\right) \\
\leq \frac{1}{3} c_{3}\left[h \sum_{i=1}^{m-1}\left(e_{i}^{k+\frac{1}{2}}\right)^{2}+\frac{1}{2} h \sum_{i=1}^{m-1}\left|e_{i+1}^{k+\frac{1}{2}} e_{i}^{k+\frac{1}{2}}\right|\right] \leq \frac{1}{2} c_{3}\left\|e^{k+\frac{1}{2}}\right\|^{2} .
\end{gathered}
$$

Using Lemma 2.3, we have

$$
\begin{aligned}
& h \sum_{i=1}^{m-2}\left(\delta_{x}^{2} \delta_{x} e_{i+\frac{1}{2}}^{k+\frac{1}{2}}\right) e_{i}^{k+\frac{1}{2}}+\frac{1}{h}\left(\delta_{x} e_{m-\frac{3}{2}}^{k+\frac{1}{2}}-3 \delta_{x} e_{m-\frac{1}{2}}^{k+\frac{1}{2}}\right) e_{m-1}^{k+\frac{1}{2}} \\
= & \frac{1}{2} h^{2} \sum_{i=1}^{m-1}\left(\delta_{x}^{2} e_{i}^{k+\frac{1}{2}}\right)^{2}+\frac{1}{2}\left(\delta_{x} e_{\frac{1}{2}}^{k+\frac{1}{2}}\right)^{2}+\frac{3}{2}\left(\delta_{x} e_{m-\frac{1}{2}}^{k+\frac{1}{2}}\right)^{2}
\end{aligned}
$$

Substituting (3.33) and (3.32) in (3.30), we get

$$
\begin{aligned}
& \frac{1}{2 \tau}\left(\left\|e^{k+1}\right\|^{2}-\left\|e^{k}\right\|^{2}\right)+\frac{1}{2} h^{2} \sum_{i=1}^{m-1}\left(\delta_{x}^{2} e_{i}^{k+\frac{1}{2}}\right)^{2}+\frac{1}{2}\left(\delta_{x} e_{\frac{1}{2}}^{k+\frac{1}{2}}\right)^{2}+\frac{3}{2}\left(\delta_{x} e_{m-\frac{1}{2}}^{k+\frac{1}{2}}\right)^{2} \\
\leq & \frac{|\gamma|}{2} c_{3}\left\|e^{k+\frac{1}{2}}\right\|^{2}+\left\|P^{k+\frac{1}{2}}\right\| \cdot\left\|e^{k+\frac{1}{2}}\right\| \\
\leq & \left(\frac{|\gamma|}{2} c_{3} \frac{\left\|e^{k+1}\right\|+\left\|e^{k}\right\|}{2}+\left\|P^{k+\frac{1}{2}}\right\|\right) \frac{\left\|e^{k+1}\right\|+\left\|e^{k}\right\|}{2}, \quad 0 \leq k \leq n-1 .
\end{aligned}
$$

Consequently, we have

$$
\begin{aligned}
& \frac{1}{2 \tau}\left(\left(F^{k+1}\right)^{2}-\left(F^{k}\right)^{2}\right) \\
\leq & \left(\frac{|\gamma|}{2} c_{3} \frac{F^{k+1}+F^{k}}{2}+\left\|P^{k+\frac{1}{2}}\right\|\right) \frac{F^{k+1}+F^{k}}{2}, \quad 0 \leq k \leq n-1 .
\end{aligned}
$$

Crossing out $\frac{1}{2}\left(F^{k+1}+F^{k}\right)$ from both sides, we have

$$
\frac{1}{\tau}\left(F^{k+1}-F^{k}\right) \leq \frac{1}{2}|\gamma| c_{3} \frac{F^{k+1}+F^{k}}{2}+\left\|P^{k+\frac{1}{2}}\right\|, \quad 0 \leq k \leq n-1 .
$$

Noticing (3.4) and (3.9), we have

$$
\begin{aligned}
& \left(1-\frac{|\gamma| c_{3}}{4} \tau\right) F^{k+1} \\
\leq & \left(1+\frac{|\gamma| c_{3}}{4} \tau\right) F^{k}+\sqrt{L\left(c_{0}^{2}+c_{1}^{2}\right)} \tau\left(h+\tau^{2}\right), \quad 0 \leq k \leq n-1 .
\end{aligned}
$$

If $\gamma=0$, it is easy to obtain

$$
F^{k+1} \leq \sqrt{L\left(c_{0}^{2}+c_{1}^{2}\right)} T\left(h+\tau^{2}\right), \quad 0 \leq k \leq n-1 .
$$


When $\gamma \neq 0$ and $\frac{|\gamma| c_{3}}{4} \tau \leq \frac{1}{3}$, it follows

$$
F^{k+1} \leq\left(1+\frac{3|\gamma| c_{3}}{4} \tau\right) F^{k}+\frac{3}{2} \sqrt{L\left(c_{0}^{2}+c_{1}^{2}\right)} \tau\left(h+\tau^{2}\right), \quad 0 \leq k \leq n-1 .
$$

By Gronwall inequality, we obtain

$$
F^{k+1} \leq e^{\frac{3|\gamma| c_{3}}{4} T} \frac{2 \sqrt{L\left(c_{0}^{2}+c_{1}^{2}\right)}}{|\gamma| c_{3}}\left(h+\tau^{2}\right), \quad 0 \leq k \leq n-1 .
$$

This completes the proof.

Remark 3.3. From the convergence result in Theorem 3.5, we know that the $E^{k}$ defined in Theorem 3.3 is a simulation of the energy $E\left(t_{k}\right)$ defined in Lemma 2.7.

\section{A three-level linearized finite difference scheme}

In this section, we first present a three-level linearized finite difference scheme for (1.1a)-(1.1c) and then prove its existence, boundeness and convergence of the proposed scheme.

\subsection{The derivation of the finite difference scheme}

Considering (1.1a) at point $\left(x_{i}, t_{0}\right)$ and noticing initial value conditions, we have

$$
\begin{aligned}
u_{t}\left(x_{i}, t_{0}\right) & =-\gamma u\left(x_{i}, t_{0}\right) u_{x}\left(x_{i}, t_{0}\right)-u_{x x x}\left(x_{i}, t_{0}\right) \\
& =-\gamma \varphi\left(x_{i}\right) \varphi^{\prime}\left(x_{i}\right)-\varphi^{\prime \prime \prime}\left(x_{i}\right), \quad 0 \leq i \leq m .
\end{aligned}
$$

Set

$$
\hat{u}=\varphi\left(x_{i}\right)+\frac{\tau}{2}\left[-\gamma \varphi\left(x_{i}\right) \varphi^{\prime}\left(x_{i}\right)-\varphi^{\prime \prime \prime}\left(x_{i}\right)\right], \quad 0 \leq i \leq m .
$$

Considering Eq. (1.1a) at the points $\left(x_{i}, t_{\frac{1}{2}}\right)$, and using the Lemma 2.3, Lemma 2.4, Lemma 2.1 and (3.6), we have

$$
\begin{aligned}
& \delta_{t} U_{i}^{\frac{1}{2}}+\gamma \psi\left(\hat{u}, U^{\frac{1}{2}}\right)_{i}+\delta_{x}^{2} \delta_{x} U_{i+\frac{1}{2}}^{\frac{1}{2}}=Q_{i}^{0}, \quad 1 \leq i \leq m-2, \\
& \delta_{t} U_{m-1}^{\frac{1}{2}}+\gamma \psi\left(\hat{u}, U^{\frac{1}{2}}\right)_{m-1}+\frac{1}{h^{2}}\left(\delta_{x} U_{m-\frac{3}{2}}^{\frac{1}{2}}-3 \delta_{x} U_{m-\frac{1}{2}}^{\frac{1}{2}}\right)=Q_{m-1}^{0}
\end{aligned}
$$

Considering Eq. (1.1a) at the points $\left(x_{i}, t_{k}\right)$ and using the Taylor expansion, we get

$$
\begin{aligned}
& \Delta_{t} U_{i}^{k}+\gamma \psi\left(U^{k}, U^{\bar{k}}\right)_{i}+\delta_{x}^{2} \delta_{x} U_{i+\frac{1}{2}}^{\bar{k}}=Q_{i}^{k}, \quad 1 \leq i \leq m-2, \quad 1 \leq k \leq n-1, \\
& \Delta_{t} U_{m-1}^{k}+\gamma \psi\left(U^{k}, U^{\bar{k}}\right)_{m-1}+\frac{1}{h^{2}}\left(\delta_{x} U_{m-\frac{3}{2}}^{\bar{k}}-3 \delta_{x} U_{m-\frac{1}{2}}^{\bar{k}}\right) \\
& =Q_{m-1}^{k}, \quad 1 \leq k \leq n-1 .
\end{aligned}
$$


There exists a constant $c_{4}>0$ such that

$$
\left|Q_{i}^{k}\right| \leq c_{4}\left(h+\tau^{2}\right), \quad 1 \leq i \leq m-1, \quad 0 \leq k \leq n-1 .
$$

Noticing the initial and boundary value conditions (1.1b)-(1.1c)

$$
\begin{array}{ll}
U_{i}^{0}=\varphi\left(x_{i}\right), & 1 \leq i \leq m-1, \\
U_{0}^{k}=0, \quad U_{m}^{k}=0, & 0 \leq k \leq n,
\end{array}
$$

and omitting the small terms in (4.2a)-(4.2b) and (4.3a)-(4.3b), we construct a linearized finite difference scheme for the problem (1.1a)-(1.1c) as follows:

$$
\begin{array}{ll}
\delta_{t} u_{i}^{\frac{1}{2}}+\gamma \psi\left(\hat{u}, u^{\frac{1}{2}}\right)_{i}+\delta_{x}^{2}\left(\delta_{x} u_{i+\frac{1}{2}}^{\frac{1}{2}}\right)=0, & 1 \leq i \leq m-2, \\
\delta_{t} u_{m-1}^{\frac{1}{2}}+\gamma \psi\left(\hat{u}, u^{\frac{1}{2}}\right)_{m-1}+\frac{1}{h^{2}}\left(\delta_{x} u_{m-\frac{3}{2}}^{\frac{1}{2}}-3 \delta_{x} u_{m-\frac{1}{2}}^{\frac{1}{2}}\right)=0, & \\
\Delta_{t} u_{i}^{k}+\gamma \psi\left(u^{k}, u^{\bar{k}}\right)_{i}+\delta_{x}^{2}\left(\delta_{x} u_{i+\frac{1}{2}}^{\bar{k}}\right)=0, \quad 1 \leq i \leq m-2, \quad 1 \leq k \leq n-1, \\
\Delta_{t} u_{m-1}^{k}+\gamma \psi\left(u^{k}, u^{\bar{k}}\right)_{m-1}+\frac{1}{h^{2}}\left(\delta_{x} u_{m-\frac{3}{2}}^{\bar{k}}-3 \delta_{x} u_{m-\frac{1}{2}}^{\bar{k}}\right)=0, \quad 1 \leq k \leq n-1, \\
u_{i}^{0}=\varphi\left(x_{i}\right), \quad 1 \leq i \leq m-1, \\
u_{0}^{k}=0, \quad u_{m}^{k}=0, \quad 0 \leq k \leq n .
\end{array}
$$

\subsection{Existence and boundedness of the difference solution}

Theorem 4.1. The finite difference scheme (4.6a)-(4.6f) has a unique solution.

Proof. By (4.6e) and (4.6f), $u^{0}$ is given. Using (4.6a)-(4.6b), we solve a linear system of algebraic equations about $u^{1}$. Consider its homogenous equations

$$
\begin{aligned}
& \frac{1}{\tau} u_{i}^{1}+\frac{1}{2} \gamma \psi\left(\hat{u}, u^{1}\right)_{i}+\frac{1}{2} \delta_{x}^{2} \delta_{x} u_{i+\frac{1}{2}}^{1}=0, \quad 1 \leq i \leq m-2, \\
& \frac{1}{\tau} u_{m-1}^{1}+\frac{1}{2} \gamma \psi\left(\hat{u}, u^{1}\right)_{m-1}+\frac{1}{2} \cdot \frac{1}{h^{2}}\left(\delta_{x} u_{m-\frac{3}{2}}^{1}-3 \delta_{x} u_{m-\frac{1}{2}}^{1}\right)=0 \\
& u_{0}^{1}=0, \quad u_{m}^{1}=0 .
\end{aligned}
$$

Multiplying (4.7a) and (4.7b) by $h u_{i}^{1}$ and $h u_{m-1}^{1}$, and summing up the results, we have

$$
\begin{aligned}
\frac{1}{\tau}\left\|u^{1}\right\|^{2} & +\frac{1}{2} \gamma\left(\psi\left(\hat{u}, u^{1}\right), u^{1}\right) \\
& +\frac{1}{2}\left[h \sum_{i=1}^{m-2}\left(\delta_{x}^{2} \delta_{x} u_{i+\frac{1}{2}}^{1}\right) u_{i}^{1}+\frac{1}{h}\left(\delta_{x} u_{m-\frac{3}{2}}^{1}-3 \delta_{x} u_{m-\frac{1}{2}}^{1}\right) u_{m-1}^{1}\right]=0
\end{aligned}
$$

Owing to Lemma 2.3 and Lemma 2.1, we have

$$
\left\|u^{1}\right\|^{2} \leq 0 .
$$


Therefore $u^{1}$ is uniquely determined. Suppose $u^{k-1}$ and $u^{k}$ have been determined. Then we can obtain the linear system of algebraic equations about $u^{k+1}$ from (4.6c), (4.6d) and (4.6f). Consider its homogeneous equations

$$
\begin{aligned}
& \frac{1}{2 \tau} u_{i}^{k+1}+\frac{1}{2} \gamma \psi\left(u^{k}, u^{k+1}\right)_{i}+\frac{1}{2} \delta_{x}^{2}\left(\delta_{x} u_{i+\frac{1}{2}}^{k+1}\right)=0, \quad 1 \leq i \leq m-2, \\
& \frac{1}{2 \tau} u_{m-1}^{k+1}+\frac{1}{2} \gamma \psi\left(u^{k}, u^{k+1}\right)_{m-1}+\frac{1}{2} \cdot \frac{1}{h^{2}}\left(\delta_{x} u_{m-\frac{3}{2}}^{k+1}-3 \delta_{x} u_{m-\frac{1}{2}}^{k+1}\right)=0, \\
& u_{0}^{k+1}=0, \quad u_{m}^{k+1}=0 .
\end{aligned}
$$

Multiplying (4.8a) and (4.8b) by $2 h u_{i}^{k+1}$ and $2 h u_{m-1}^{k+1}$, and summing up the results, we get

$$
\begin{gathered}
\frac{1}{\tau}\left\|u^{k+1}\right\|^{2}+\gamma\left(\psi\left(u^{k}, u^{k+1}\right), u^{k+1}\right)+h \sum_{i=2}^{m-1}\left(\delta_{x}^{2} \delta_{x} u_{i+\frac{1}{2}}^{k+1}\right) u_{i}^{k+1} \\
+\frac{1}{h}\left(\delta_{x} u_{m-\frac{3}{2}}^{k+1}-3 \delta_{x} u_{m-\frac{1}{2}}^{k+1}\right) u_{m-1}^{k+1}=0 .
\end{gathered}
$$

Using Lemma 2.3 and Lemma 2.1, we have

$$
\left\|u^{k+1}\right\|^{2} \leq 0
$$

This means that (4.6c), (4.6d) and (4.6f) determine $u^{k+1}$ uniquely. This completes the proof.

Theorem 4.2. Suppose $\left\{u_{i}^{k} \mid 0 \leq i \leq m, 0 \leq k \leq n\right\}$ be the solution of the three-level linearized finite difference scheme (4.6a)-(4.6f). Denote

$$
\begin{gathered}
\hat{E}^{k}=\frac{1}{2}\left(\left\|u^{k+1}\right\|^{2}+\left\|u^{k}\right\|^{2}\right)+\tau \sum_{l=1}^{k}\left[\left(\delta_{x} u_{\frac{1}{2}}^{\bar{l}}\right)^{2}+3\left(\delta_{x} u_{m-\frac{1}{2}}^{\bar{l}}\right)^{2}+h^{2} \sum_{i=1}^{m-1}\left(\delta_{x}^{2} u_{i}^{\bar{l}}\right)^{2}\right] \\
+\frac{\tau}{2}\left[\left(\delta_{x} u_{\frac{1}{2}}^{\frac{1}{2}}\right)^{2}+3\left(\delta_{x} u_{m-\frac{1}{2}}^{\frac{1}{2}}\right)^{2}+h^{2} \sum_{i=1}^{m-1}\left(\delta_{x}^{2} u_{i}^{\frac{1}{2}}\right)^{2}\right] .
\end{gathered}
$$

Then

$$
\hat{E}^{k}=\left\|u^{0}\right\|^{2}, \quad 0 \leq k \leq n-1
$$

Proof. Multiplying (4.6a)-(4.6b) by $h u_{i}^{\frac{1}{2}}$ and $h u_{m-1}^{\frac{1}{2}}$, and summing up the results, we get

$$
\begin{gathered}
\frac{1}{2 \tau}\left(\left\|u^{1}\right\|^{2}-\left\|u^{0}\right\|^{2}\right)+\gamma\left(\psi\left(\hat{u}, u^{\frac{1}{2}}\right), u^{\frac{1}{2}}\right)+h \sum_{i=1}^{m-2}\left(\delta_{x}^{2} \delta_{x} u_{i+\frac{1}{2}}^{\frac{1}{2}}\right) u_{i}^{\frac{1}{2}} \\
+\frac{1}{h}\left(\delta_{x} u_{m-\frac{3}{2}}^{\frac{1}{2}}-3 \delta_{x} u_{m-\frac{1}{2}}^{\frac{1}{2}}\right) u_{m-1}^{\frac{1}{2}}=0 .
\end{gathered}
$$


Since $\left(\psi\left(\hat{u}, u^{\frac{1}{2}}\right), u^{\frac{1}{2}}\right)=0$ and using Lemma 2.3, we have

$$
\frac{1}{2 \tau}\left(\left\|u^{1}\right\|^{2}-\left\|u^{0}\right\|^{2}\right)+\frac{1}{2} h^{2} \sum_{i=1}^{m-1}\left(\delta_{x}^{2} u_{i}^{\frac{1}{2}}\right)^{2}+\frac{1}{2}\left(\delta_{x} u_{\frac{1}{2}}^{\frac{1}{2}}\right)^{2}+\frac{3}{2}\left(\delta_{x} u_{m-\frac{1}{2}}^{\frac{1}{2}}\right)^{2}=0,
$$

or

$$
\frac{1}{2}\left(\left\|u^{1}\right\|^{2}+\left\|u^{0}\right\|^{2}\right)+\frac{\tau}{2}\left[h^{2} \sum_{i=1}^{m-1}\left(\delta_{x}^{2} u_{i}^{\frac{1}{2}}\right)^{2}+\left(\delta_{x} u_{\frac{1}{2}}^{\frac{1}{2}}\right)^{2}+3\left(\delta_{x} u_{m-\frac{1}{2}}^{\frac{1}{2}}\right)^{2}\right]=\left\|u^{0}\right\|^{2} .
$$

Multiplying (4.6c) and (4.6d) by $h u_{i}^{\bar{k}}$ and $h u_{m-1}^{\bar{k}}$ and summing up the results, we get

$$
\begin{gathered}
\frac{1}{4 \tau}\left(\left\|u^{k+1}\right\|^{2}-\left\|u^{k-1}\right\|^{2}\right)+\gamma\left(\psi\left(u^{k}, u^{\bar{k}}\right), u^{\bar{k}}\right)+h \sum_{i=1}^{m-2}\left(\delta_{x}^{2} \delta_{x} u_{i+\frac{1}{2}}^{\bar{k}}\right) u_{i}^{\bar{k}} \\
+\frac{1}{2}\left(\delta_{x} u_{m-\frac{3}{2}}^{\bar{k}}-3 \delta_{x} u_{m-\frac{1}{2}}^{\bar{k}}\right) u_{m-1}^{\bar{k}}=0, \quad 1 \leq k \leq n-1 .
\end{gathered}
$$

Applying Lemma 2.3 and noticing $\left(\psi\left(u^{k}, u^{\bar{k}}\right), u^{\bar{k}}\right)=0$, we get

$$
\begin{aligned}
\frac{1}{4 \tau}\left(\left\|u^{k+1}\right\|^{2}-\left\|u^{k-1}\right\|^{2}\right)+\frac{1}{2} h^{2} \sum_{i=1}^{m-1}\left(\delta_{x}^{2} u_{i}^{\bar{k}}\right)^{2} \\
\quad+\frac{1}{2}\left(\delta_{x} u_{\frac{1}{2}}^{\bar{k}}\right)^{2}+\frac{3}{2}\left(\delta_{x} u_{m-\frac{1}{2}}^{\bar{k}}\right)^{2}=0, \quad 1 \leq k \leq n-1
\end{aligned}
$$

and replacing $k$ by $l$ and summing up for $l$ from 1 to $k$, then multiplying $2 \tau$ on both sides in (4.16) and adding with (4.14), we obtain

$$
\begin{gathered}
\frac{1}{2}\left(\left\|u^{k+1}\right\|^{2}+\left\|u^{k}\right\|^{2}\right)+\tau \sum_{l=1}^{k}\left[\left(\delta_{x} u_{\frac{1}{2}}^{\bar{l}}\right)^{2}+3\left(\delta_{x} u_{m-\frac{1}{2}}^{\bar{l}}\right)^{2}+h^{2} \sum_{i=1}^{m-1}\left(\delta_{x}^{2} u_{i}^{\bar{l}}\right)^{2}\right] \\
+\frac{\tau}{2}\left[\left(\delta_{x} u_{\frac{1}{2}}^{\frac{1}{2}}\right)^{2}+3\left(\delta_{x} u_{m-\frac{1}{2}}^{\frac{1}{2}}\right)^{2}+h^{2} \sum_{i=1}^{m-1}\left(\delta_{x}^{2} u_{i}^{\frac{1}{2}}\right)^{2}\right]=\left\|u^{0}\right\|^{2}
\end{gathered}
$$

This completes this proof.

\subsection{The convergence of the finite difference scheme}

Theorem 4.3. Assume that $u(x, t) \in C^{3}\left(0, T ; C^{4}(0, L)\right)$ is the solution of the problem (1.1a)-(1.1c) and $\left\{u_{i}^{k} \mid 0 \leq i \leq m, 0 \leq k \leq n\right\}$ is the solution of finite difference scheme (4.6a)-(4.6f), respectively. Denote

$$
\begin{aligned}
& \hat{c}=\max _{0 \leq x \leq L, 0 \leq t \leq L}|u(x, t)|, \quad \lambda=\frac{|\gamma| \hat{c}}{3} \frac{\tau}{h}, \\
& e_{i}^{k}=U_{i}^{k}-u_{i}^{k}, \quad 0 \leq i \leq m, \quad 0 \leq k \leq n
\end{aligned}
$$


and

$$
\begin{aligned}
\hat{F}^{k}=\frac{1}{2}\left(\left\|e^{k+1}\right\|^{2}+\left\|e^{k}\right\|^{2}\right)+\tau \sum_{l=1}^{k}\left[\left(\delta_{x} e_{\frac{1}{2}}^{\bar{l}}\right)^{2}+3\left(\delta_{x} e_{m-\frac{1}{2}}^{\bar{l}}\right)^{2}+h^{2} \sum_{i=1}^{m-1}\left(\delta_{x}^{2} e_{i}^{\bar{l}}\right)^{2}\right] \\
+\frac{\tau}{2}\left[\left(\delta_{x} e_{\frac{1}{2}}^{\frac{1}{2}}\right)^{2}+3\left(\delta_{x} e_{m-\frac{1}{2}}^{\frac{1}{2}}\right)^{2}+h^{2} \sum_{i=1}^{m-1}\left(\delta_{x}^{2} e_{i}^{\frac{1}{2}}\right)^{2}\right], \quad 0 \leq k \leq n-1 .
\end{aligned}
$$

Then, if $\lambda<1$, there exists a positive constant $c_{5}$ such that

$$
\hat{F}^{k} \leq c_{5}\left(h+\tau^{2}\right)^{2}, \quad 0 \leq k \leq n-1 .
$$

Proof. Let

$$
c_{6}=\max _{0 \leq x \leq L, 0 \leq t \leq T}\left|u_{t}(x, t)\right| .
$$

Subtracting (4.6a)-(4.6f) from (4.2a)-(4.3b) and (4.5a)-(4.5b), we obtain error equations

$$
\begin{aligned}
& \delta_{t} e_{i}^{\frac{1}{2}}+\gamma \psi\left(\hat{u}, e^{\frac{1}{2}}\right)_{i}+\delta_{x}^{2}\left(\delta_{x} e_{i+\frac{1}{2}}^{\frac{1}{2}}\right)=Q_{i}^{0}, \quad 1 \leq i \leq m-2, \\
& \delta_{t} e_{m-1}^{\frac{1}{2}}+\gamma \psi\left(\hat{u}, e^{\frac{1}{2}}\right)_{m-1}+\frac{1}{h^{2}}\left(\delta_{x} e_{m-\frac{3}{2}}^{\frac{1}{2}}-3 \delta_{x} e_{m-\frac{1}{2}}^{\frac{1}{2}}\right)=Q_{m-1}^{0}, \\
& \Delta_{t} e_{i}^{k}+\gamma\left[\psi\left(U^{k}, U^{\bar{k}}\right)_{i}-\psi\left(u^{k}, u^{\bar{k}}\right)_{i}\right]+\delta_{x}^{2}\left(\delta_{x} e_{i+\frac{1}{2}}^{\bar{k}}\right) \\
& =Q_{i}^{k}, \\
& 1 \leq i \leq m-2, \quad 1 \leq k \leq n-1, \\
& \Delta_{t} e_{m-1}^{k}+\gamma\left[\psi\left(U^{k}, U^{\bar{k}}\right)_{m-1}-\psi\left(u^{k}, u^{\bar{k}}\right)_{m-1}\right]+\frac{1}{h^{2}}\left(\delta_{x} e_{m-\frac{3}{2}}^{\bar{k}}-3 \delta_{x} e_{m-\frac{1}{2}}^{\bar{k}}\right) \\
& =Q_{m-1}^{k}, \quad 1 \leq k \leq n-1, \\
& e_{i}^{0}=0, \\
& e_{0}^{k}=0, \quad e_{m}^{k}=0, \quad 0 \leq k \leq n
\end{aligned}
$$

Multiplying (4.20a) by $h e_{i}^{\frac{1}{2}}$, multiplying (4.20b) by $h e_{m-1}^{\frac{1}{2}}$ and adding the results, we get

$$
\begin{gathered}
\left(\delta_{t} e^{\frac{1}{2}}, e^{\frac{1}{2}}\right)+\gamma\left(\psi\left(\hat{u}, e^{\frac{1}{2}}\right), e^{\frac{1}{2}}\right)+\left[h \sum_{i=1}^{m-2}\left(\delta_{x}^{2} \delta_{x} e_{i+\frac{1}{2}}^{\frac{1}{2}}\right) e_{i}^{\frac{1}{2}}\right. \\
\left.+\frac{1}{h}\left(\delta_{x} e_{m-\frac{3}{2}}^{\frac{1}{2}}-3 \delta_{x} e_{m-\frac{1}{2}}^{\frac{1}{2}}\right) e_{m-1}^{\frac{1}{2}}\right]=\left(Q^{0}, e^{\frac{1}{2}}\right)
\end{gathered}
$$

By Lemma 2.1 and Lemma 2.3, we obtain

$$
\left(\delta_{t} e^{\frac{1}{2}}, e^{\frac{1}{2}}\right)+\frac{1}{2}\left[h^{2} \sum_{i=1}^{m-1}\left(\delta_{x}^{2} e_{i}^{\frac{1}{2}}\right)^{2}+\left(\delta_{x} e_{\frac{1}{2}}^{\frac{1}{2}}\right)^{2}+3\left(\delta_{x} e_{m-\frac{1}{2}}^{\frac{1}{2}}\right)^{2}\right]=\left(Q^{0}, e^{\frac{1}{2}}\right) .
$$


Noticing (4.20e) and (4.20f), we get

$$
\begin{aligned}
& \frac{1}{2 \tau}\left\|e^{1}\right\|^{2}+\frac{1}{2}\left[h^{2} \sum_{i=1}^{m-1}\left(\delta_{x}^{2} e_{i}^{\frac{1}{2}}\right)^{2}+\left(\delta_{x} e_{\frac{1}{2}}^{\frac{1}{2}}\right)^{2}+3\left(\delta_{x} e_{m-\frac{1}{2}}^{\frac{1}{2}}\right)^{2}\right] \\
= & \frac{1}{2}\left(Q^{0}, e^{1}\right) \leq \frac{1}{4 \tau}\left\|e^{1}\right\|^{2}+\frac{\tau}{4}\left\|Q^{0}\right\|^{2},
\end{aligned}
$$

which follows that

$$
\hat{F}^{0} \leq \frac{\tau}{2}\left\|Q^{0}\right\|^{2} \leq \frac{\tau}{2} L c_{4}^{2}\left(h+\tau^{2}\right)^{2} \leq \frac{T}{2} L c_{4}^{2}\left(h+\tau^{2}\right)^{2} .
$$

Multiplying (4.20c) by $h e_{i}^{\bar{k}}$, multiplying (4.20d) by $h e_{m-1}^{\bar{k}}$, summing up the results, we have

$$
\begin{aligned}
& \left(\Delta_{t} e^{k}, e^{\bar{k}}\right)+\gamma\left(\psi\left(U^{k}, U^{\bar{k}}\right)-\psi\left(u^{k}, u^{\bar{k}}\right), e^{\bar{k}}\right) \\
& +\left[h \sum_{i=1}^{m-2}\left(\delta_{x}^{2} \delta_{x} e_{i+\frac{1}{2}}^{\bar{k}}\right) e_{i}^{\bar{k}}+\frac{1}{h}\left(\delta_{x} e_{m-\frac{3}{2}}^{\bar{k}}-3 \delta_{x} e_{m-\frac{1}{2}}^{\bar{k}}\right) e_{m-1}^{\bar{k}}\right] \\
= & \left(Q^{k}, e^{\bar{k}}\right), \quad 1 \leq k \leq n-1 .
\end{aligned}
$$

It is easy to know that

$$
\begin{aligned}
\left(\Delta_{t} e^{k}, e^{\bar{k}}\right) & =\frac{1}{4 \tau}\left(\left\|e^{k+1}\right\|^{2}-\left\|e^{k-1}\right\|^{2}\right) \\
& =\frac{1}{2 \tau}\left(\frac{\left\|e^{k+1}\right\|^{2}+\left\|e^{k}\right\|^{2}}{2}-\frac{\left\|e^{k}\right\|^{2}+\left\|e^{k-1}\right\|^{2}}{2}\right) .
\end{aligned}
$$

Using Lemma 2.3, we have

$$
\begin{aligned}
& h \sum_{i=1}^{m-2}\left(\delta_{x}^{2} \delta_{x} e_{i+\frac{1}{2}}^{\bar{k}}\right) e_{i}^{\bar{k}}+\frac{1}{h}\left(\delta_{x} e_{m-\frac{3}{2}}^{\bar{k}}-3 \delta_{x} e_{m-\frac{1}{2}}^{\bar{k}}\right) e_{m-1}^{\bar{k}} \\
= & \frac{1}{2} h^{2} \sum_{i=1}^{m-1}\left(\delta_{x}^{2} e_{i}^{\bar{k}}\right)^{2}+\frac{1}{2}\left(\delta_{x} e_{\frac{1}{2}}^{\bar{k}}\right)^{2}+\frac{3}{2}\left(\delta_{x} e_{m-\frac{1}{2}}^{\bar{k}}\right)^{2} .
\end{aligned}
$$

By Lemma 2.3, we have

$$
\begin{aligned}
& \left(\psi\left(U^{k}, U^{\bar{k}}\right)-\psi\left(u^{k}, u^{\bar{k}}\right), e^{\bar{k}}\right)=\left(\psi\left(U^{k}, e^{\bar{k}}\right)+\psi\left(e^{k}, U^{\bar{k}}\right)-\psi\left(e^{k}, e^{\bar{k}}\right), e^{\bar{k}}\right) \\
= & \left(\psi\left(e^{k}, U^{\bar{k}}\right), e^{\bar{k}}\right)=\frac{1}{3} h \sum_{i=1}^{m-1}\left[e_{i}^{k} \Delta_{x} U_{i}^{\bar{k}}+\Delta_{x}\left(e^{k} U^{\bar{k}}\right)_{i}\right] e_{i}^{\bar{k}} \\
= & \frac{1}{3} h \sum_{i=1}^{m-1}\left(\Delta_{x} U_{i}^{\bar{k}}\right) e_{i}^{k} e_{i}^{\bar{k}}-\frac{1}{3} h \sum_{i=1}^{m-1} U_{i}^{\bar{k}} e_{i}^{k} \Delta_{x} e_{i}^{\bar{k}} \\
= & \frac{1}{3} h \sum_{i=1}^{m-1}\left(\Delta_{x} U_{i}^{\bar{k}}\right) e_{i}^{k} e_{i}^{\bar{k}}+\frac{1}{6} \sum_{i=1}^{m-2}\left(U_{i+1}^{\bar{k}} e_{i+1}^{k} e_{i}^{\bar{k}}-U_{i}^{\bar{k}} e_{i}^{k} e_{i+1}^{\bar{k}}\right)
\end{aligned}
$$




$$
\begin{aligned}
& =\frac{1}{3} h \sum_{i=1}^{m-1}\left(\Delta_{x} U_{i}^{\bar{k}}\right) e_{i}^{k} e_{i}^{\bar{k}}+\frac{1}{6} \sum_{i=1}^{m-2}\left(U_{i+1}^{\bar{k}}-U_{i}^{\bar{k}}\right) e_{i+1}^{k} e_{i}^{\bar{k}}+\frac{1}{6} \sum_{i=1}^{m-2} U_{i}^{\bar{k}}\left(e_{i}^{\bar{k}} e_{i+1}^{k}-e_{i}^{k} e_{i+1}^{\bar{k}}\right) \\
& =\frac{1}{3} h \sum_{i=1}^{m-1}\left(\Delta_{x} U_{i}^{\bar{k}}\right) e_{i}^{k} e_{i}^{\bar{k}}+\frac{1}{6} h \sum_{i=1}^{m-2}\left(\delta_{x} U_{i+\frac{1}{2}}^{\bar{k}}\right) e_{i+1}^{k} e_{i}^{\bar{k}} \\
& \quad+\frac{1}{12} \sum_{i=1}^{m-2} U_{i}^{\bar{k}}\left[\left(e_{i}^{k+1} e_{i+1}^{k}-e_{i}^{k} e_{i+1}^{k+1}\right)-\left(e_{i}^{k} e_{i+1}^{k-1}-e_{i}^{k-1} e_{i+1}^{k}\right)\right] \\
& =\frac{1}{12}\left[\sum_{i=1}^{m-2} U_{i}^{k+\frac{1}{2}}\left(e_{i}^{k+1} e_{i+1}^{k}-e_{i}^{k} e_{i+1}^{k+1}\right)-\sum_{i=1}^{m-2} U_{i}^{k-\frac{1}{2}}\left(e_{i}^{k} e_{i+1}^{k-1}-e_{i}^{k-1} e_{i+1}^{k}\right)\right] \\
& \quad+\frac{1}{3} h \sum_{i=1}^{m-1}\left(\Delta_{x} U_{i}^{\bar{k}}\right) e_{i}^{k} e_{i}^{\bar{k}}+\frac{1}{6} h \sum_{i=1}^{m-2}\left(\delta_{x} U_{i+\frac{1}{2}}^{\bar{k}}\right) e_{i+1}^{k} e_{i}^{\bar{k}} \\
& \quad+\frac{1}{12} \sum_{i=1}^{m-2}\left(U_{i}^{\bar{k}}-U_{i}^{k+\frac{1}{2}}\right)\left(e_{i}^{k+1} e_{i+1}^{k}-e_{i}^{k} e_{i+1}^{k+1}\right) \\
& \quad+\frac{1}{12} \sum_{i=1}^{m-1}\left(U_{i}^{k-\frac{1}{2}}-U_{i}^{\bar{k}}\right)\left(e_{i}^{k} e_{i+1}^{k-1}-e_{i}^{k-1} e_{i+1}^{k}\right) .
\end{aligned}
$$

Denote

$$
G^{k}=\frac{\gamma}{6} \tau \sum_{i=1}^{m-2} U_{i}^{k+\frac{1}{2}}\left(e_{i}^{k+1} e_{i+1}^{k}-e_{i}^{k} e_{i+1}^{k-1}\right) .
$$

By Lemma 2.1, substituting (4.24)-(4.26) into (4.23), we have

$$
\begin{aligned}
& \frac{1}{2 \tau}\left[\left(\hat{F}^{k}+G^{k}\right)-\left(\hat{F}^{k-1}+G^{k-1}\right)\right] \\
& \leq-\gamma\left\{\frac{1}{3} h \sum_{i=1}^{m-1}\left(\Delta_{x} U_{i}^{\bar{k}}\right) e_{i}^{k} e_{i}^{\bar{k}}+\frac{1}{6} h \sum_{i=1}^{m-2}\left(\delta_{x} U_{i+\frac{1}{2}}^{\bar{k}}\right) e_{i+1}^{k} e_{i}^{\bar{k}}\right. \\
& +\frac{1}{12} \sum_{i=1}^{m-2}\left(U_{i}^{k-1}-U_{i}^{k}\right)\left(e_{i}^{k+1} e_{i+1}^{k}-e_{i}^{k} e_{i+1}^{k+1}\right) \\
& \left.\quad+\frac{1}{12} \sum_{i=1}^{m-2}\left(U_{i}^{k}-U_{i}^{k+1}\right)\left(e_{i}^{k} e_{i+1}^{k-1}-e_{i}^{k-1} e_{i+1}^{k}\right)\right\}+\left(Q^{k}, e^{\bar{k}}\right) \\
& \leq|\gamma|\left(\frac{1}{2} c_{3}\left\|e^{k}\right\| \cdot\left\|e^{\bar{k}}\right\|+\frac{1}{6} c_{6} \frac{\tau}{h}\left\|e^{k}\right\| \cdot\left\|e^{k+1}\right\|+\frac{1}{6} c_{6} \frac{\tau}{h}\left\|e^{k}\right\| \cdot\left\|e^{k-1}\right\|\right)+\left\|Q^{k}\right\| \cdot\left\|e^{\bar{k}}\right\| \\
& \leq \frac{1}{2}|\gamma| c_{3}\left\|e^{k}\right\|\left\|e^{\bar{k}}\right\|+\frac{1}{6}|\gamma| c_{6} \frac{\tau}{h}\left(\frac{\left\|e^{k}\right\|^{2}+\left\|e^{k+1}\right\|^{2}}{2}+\frac{\left\|e^{k}\right\|^{2}+\left\|e^{k-1}\right\|^{2}}{2}\right) \\
& \quad+\frac{1}{2}\left(\left\|Q^{k}\right\|^{2}+\left\|e^{\bar{k}}\right\|^{2}\right), \quad 1 \leq k \leq n-1 .
\end{aligned}
$$


It is easy to know

$$
\begin{aligned}
\left|G^{k}\right| & \leq \frac{|\gamma|}{6} \frac{c_{0} \tau}{h} h \sum_{i=1}^{m-2}\left(\left|e_{i}^{k+1} e_{i+1}^{k}\right|+\left|e_{i}^{k} e_{i+1}^{k+1}\right|\right) \\
& \leq \frac{c_{0}|\gamma|}{3} \frac{\tau}{h} \frac{\left\|e^{k+1}\right\|^{2}+\left\|e^{k}\right\|^{2}}{2}=\lambda \frac{\left\|e^{k+1}\right\|^{2}+\left\|e^{k}\right\|^{2}}{2} .
\end{aligned}
$$

On the other hand, denote

$$
H^{k}=\hat{F}^{k}+G^{k}
$$

we have

$$
H^{k} \geq \hat{F}^{k}-\lambda \frac{\left\|e^{k+1}\right\|^{2}+\left\|e^{k}\right\|^{2}}{2} \geq(1-\lambda) \hat{F}^{k}
$$

and

$$
H^{k} \geq(1-\lambda) \frac{\left\|e^{k+1}\right\|^{2}+\left\|e^{k}\right\|^{2}}{2} .
$$

By (4.27) and (4.29), there exists a constant $c_{7}$

$$
\frac{1}{2 \tau}\left(H^{k}-H^{k-1}\right) \leq \frac{1}{2} c_{7}\left(H^{k}+H^{k-1}\right)+\frac{1}{2}\left\|Q^{k}\right\|^{2}, \quad 1 \leq k \leq n-1 .
$$

Hence, we can obtain

$$
\left(1-c_{7} \tau\right) H^{k} \leq\left(1+c_{7} \tau\right) H^{k-1}+\tau\left\|Q^{k}\right\|^{2}, \quad 1 \leq k \leq n-1 .
$$

When $c_{7} \tau \leq \frac{1}{3}$,

$$
\begin{aligned}
H^{k} & \leq\left(1+3 c_{7} \tau\right) H^{k-1}+\frac{3}{2} \tau\left\|Q^{k}\right\|^{2} \\
& \leq\left(1+3 c_{7} \tau\right) H^{k-1}+\frac{3}{2} \tau c_{4}^{2} L\left(h+\tau^{2}\right)^{2}, \quad 1 \leq k \leq n-1 .
\end{aligned}
$$

By Gronwall inequality, we have

$$
H^{k} \leq e^{3 c_{7} k \tau}\left[H^{0}+\frac{c_{4}^{2} L}{2 c_{7}}\left(h+\tau^{2}\right)^{2}\right], \quad 0 \leq k \leq n-1 .
$$

Noticing (4.22) and $G^{0}=0$, we have

$$
H^{0}=\hat{F}^{0} \leq \frac{T}{2} L c_{4}^{2}\left(h+\tau^{2}\right)^{2} .
$$

Consequently,

$$
\begin{aligned}
H^{k} & \leq e^{3 c_{7} k \tau}\left[\frac{T}{2} L c_{4}^{2}\left(h+\tau^{2}\right)^{2}+\frac{c_{4}^{2} L}{2 c_{7}}\left(h+\tau^{2}\right)^{2}\right] \\
& =e^{3 c_{7} k \tau}\left(\frac{T}{2} L c_{4}^{2}+\frac{c_{4}^{2} L}{2 c_{7}}\right)\left(h+\tau^{2}\right)^{2}, \quad 0 \leq k \leq n-1,
\end{aligned}
$$


and thus, according to (4.28), we have

$$
\hat{F}^{k} \leq \frac{1}{1-\lambda} H^{k} \leq \frac{1}{1-\lambda} e^{3 c_{7} k \tau}\left(\frac{T}{2} L c_{4}^{2}+\frac{c_{4}^{2} L}{2 c_{7}}\right)\left(h+\tau^{2}\right)^{2}, \quad 0 \leq k \leq n-1 .
$$

This completes the proof of the theorem.

Remark 4.1. From the convergence result in Theorem 4.3, we know that the $\hat{E}^{k}$ defined in Theorem 4.2 is a simulation of the energy $\frac{1}{2}\left(E\left(t_{k}\right)+E\left(t_{k+1}\right)\right)$ defined in Lemma 2.7 .

\section{Numerical examples}

Both difference schemes (3.11a)-(3.11d) and (4.6a)-(4.6f) have the second order accuracy in time. At each time level, a system of nonlinear equations must be solved for the first two-level one scheme, however, only a system of linear equations is needed to be solved for the second three-level one.

In this section, we present two numerical examples. The first scheme is solved by Gauss-Seidel-like method with $10^{-8}$ as tolerance error and the second scheme is solved by double sweep method. The numerical results illustrate the efficiency of the two finite difference schemes. Suppose $\left\{u_{i}^{k}(h, \tau) \mid 0 \leq i \leq m, 0 \leq k \leq n\right\}$ is the solution of the difference scheme (3.11a)-(3.11d) or the solution of the finite difference scheme (4.6a)-(4.6f). Denote the posterior error by

$$
\begin{aligned}
& E(h, \tau)=\max _{1 \leq k \leq n}\left\{\sqrt{h \sum_{i=1}^{m-1}\left(u_{i}^{k}(h, \tau)-u_{2 i}^{k}\left(\frac{h}{2}, \tau\right)\right)^{2}}\right\}, \\
& F(h, \tau)=\left\|u^{n}(h, \tau)-u^{2 n}\left(h, \frac{\tau}{2}\right)\right\| .
\end{aligned}
$$

When $\tau$ is sufficiently small, the spatial convergence order is defined by

$$
r_{h}=\log _{2}\left(\frac{E(h, \tau)}{E\left(\frac{h}{2}, \tau\right)}\right)
$$

When $h$ is sufficiently small, the temporal convergence order is defined by

$$
r_{\tau}=\log _{2}\left(\frac{F(h, \tau)}{F\left(h, \frac{\tau}{2}\right)}\right)
$$

Example 5.1. In (1.1a)-(1.1c), take $T=1, L=1, \gamma=1, \varphi(x)=x(x-1)^{3}$.

The finite difference schemes (3.11a)-(3.11d) and (4.6a)-(4.6f) will be employed to numerically solve this example. The numerical accuracy of these two difference schemes in space and in time will be verified respectively. 


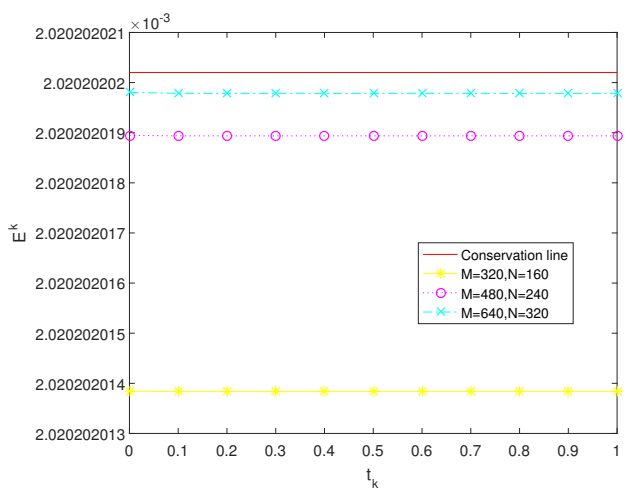

Difference schemes (3.11a)-(3.11d)

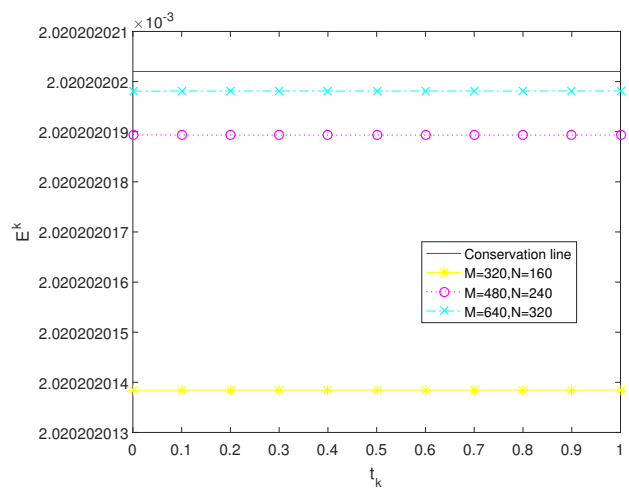

Difference schemes (4.6a)-(4.6f)

Figure 1: The energy conservation of Example 5.1.

Taking varying step size $h$ with the sufficiently small step size $\tau$, the numerical errors and convergence orders in space for schemes (3.11a)-(3.11d) and (4.6a)-(4.6f) are listed in Table 1 . Then taking varying step size $\tau$ with the sufficiently small step size $h$, the numerical errors and convergence orders in time for schemes (3.11a)-(3.11d) and (4.6a)-(4.6f) are listed in Table 2.

From these tables, we know that the numerical convergence orders of (3.11a)(3.11d) and (4.6a)-(4.6f) can achieve $\mathcal{O}\left(h+\tau^{2}\right)$, which are in a good agreement with Theorem 3.5 and Theorem 4.3. Fig. 1 indicates that the energy of the schemes (3.11a)(3.11d) and (4.6a)-(4.6f) is conserved for Example 5.1.

Table 1: Errors and convergence orders in space of Example 5.1.

\begin{tabular}{||cc|cc|cc||}
\hline \multicolumn{2}{||c|}{ Difference scheme } & \multicolumn{2}{|c|}{ (3.11a)-(3.11d) } & \multicolumn{2}{c||}{ (4.6a)-(4.6f) } \\
\hline$\tau$ & $h$ & $E(h, \tau)$ & $r_{h}$ & $E(h, \tau)$ & $r_{h}$ \\
\hline $2^{-14}$ & $2^{-5}$ & $3.213 \mathrm{e}-04$ & 0.97 & $3.213 \mathrm{e}-04$ & 0.97 \\
$2^{-14}$ & $2^{-6}$ & $1.640 \mathrm{e}-04$ & 0.98 & $1.640 \mathrm{e}-04$ & 0.98 \\
$2^{-14}$ & $2^{-7}$ & $8.298 \mathrm{e}-05$ & 0.99 & $8.299 \mathrm{e}-05$ & 0.99 \\
$2^{-14}$ & $2^{-8}$ & $4.175 \mathrm{e}-05$ & & $4.176 \mathrm{e}-05$ & \\
$2^{-14}$ & $2^{-9}$ & & & & \\
\hline
\end{tabular}

Table 2: Errors and convergence orders in time of Example 5.1.

\begin{tabular}{||cc|cc|cc||}
\hline \multicolumn{2}{||c|}{ Difference scheme } & \multicolumn{2}{|c|}{$(3.11 \mathrm{a})-(3.11 \mathrm{~d})$} & \multicolumn{2}{c||}{ (4.6a)-(4.6f) } \\
\hline$h$ & $\tau$ & $F(h, \tau)$ & $r_{\tau}$ & $F(h, \tau)$ & $r_{\tau}$ \\
\hline $2^{-18}$ & $2^{-5}$ & $4.720 \mathrm{e}-04$ & 2.50 & $1.343 \mathrm{e}-03$ & 1.51 \\
$2^{-18}$ & $2^{-6}$ & $8.337 \mathrm{e}-05$ & 2.51 & $4.716 \mathrm{e}-04$ & 2.49 \\
$2^{-18}$ & $2^{-7}$ & $1.467 \mathrm{e}-05$ & 2.02 & $8.390 \mathrm{e}-05$ & 2.54 \\
$2^{-18}$ & $2^{-8}$ & $3.605 \mathrm{e}-06$ & & $1.443 \mathrm{e}-05$ & \\
$2^{-18}$ & $2^{-9}$ & & & & \\
\hline
\end{tabular}


Example 5.2. In (1.1a)-(1.1c), take $T=1, L=1, \gamma=1, \varphi(x)=(x-1) \sin (\pi x)$.

The numerical accuracy of the finite difference schemes (3.11a)-(3.11d) and (4.6a)(4.6f) will be examined. Numerical results of these schemes under different step sizes in space and time will be calculated. And the numerical errors, as well as the convergence orders will be recorded in Tables 3-4. From these tables, we can find that the numerical results are in accord with the expected ones. Fig. 2 shows that the energy is also conserved for Example 5.2.

From the tables, we know that the numerical convergence order of (3.11a)-(3.11d) and (4.6a)-(4.6f) can achieve $\mathcal{O}\left(h+\tau^{2}\right)$, which are in a good agreement with Theorem 3.5 and Theorem 4.3. From the last columns at Tables 1-4, we see that the finite differ-

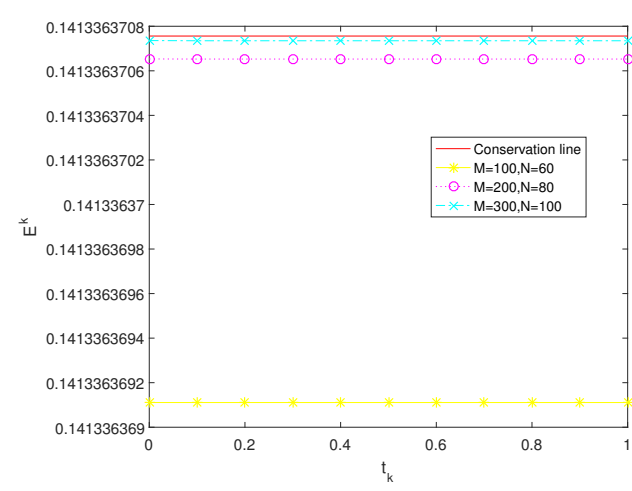

Difference schemes (3.11a)-(3.11d)

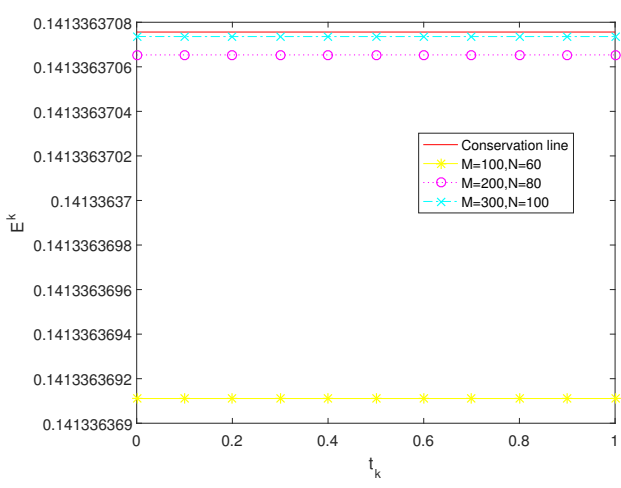

Difference schemes (4.6a)-(4.6f)

Figure 2: The energy conservation of Example 5.2.

Table 3: Error and convergence order in space of Example 5.2.

\begin{tabular}{||cc|cc|cc||}
\hline \multicolumn{2}{|c|}{ Difference scheme } & \multicolumn{2}{|c|}{ (3.11a)-(3.11d) } & \multicolumn{2}{c||}{ (4.6a)-(4.6f) } \\
\hline$\tau$ & $h$ & $E(h, \tau)$ & $r_{h}$ & $E(h, \tau)$ & $r_{h}$ \\
\hline $2^{-14}$ & $2^{-5}$ & $7.476 \mathrm{e}-03$ & 0.98 & $7.476 \mathrm{e}-03$ & 0.98 \\
$2^{-14}$ & $2^{-6}$ & $3.791 \mathrm{e}-03$ & 0.99 & $3.791 \mathrm{e}-03$ & 0.99 \\
$2^{-14}$ & $2^{-7}$ & $1.911 \mathrm{e}-03$ & 0.99 & $1.911 \mathrm{e}-03$ & 0.99 \\
$2^{-14}$ & $2^{-8}$ & $9.597 \mathrm{e}-04$ & & $9.597 \mathrm{e}-04$ & \\
$2^{-14}$ & $2^{-9}$ & & & & \\
\hline
\end{tabular}

Table 4: Error and convergence order in time of Example 5.2.

\begin{tabular}{||cc|cc|cc||}
\hline \multicolumn{2}{|c|}{ Difference scheme } & \multicolumn{2}{|c|}{ (3.11a)-(3.11d) } & \multicolumn{2}{c||}{ (4.6a)-(4.6f) } \\
\hline$h$ & $\tau$ & $F(h, \tau)$ & $r_{\tau}$ & $F(h, \tau)$ & $r_{\tau}$ \\
\hline $2^{-18}$ & $2^{-5}$ & $1.341 \mathrm{e}-02$ & 2.59 & $6.842 \mathrm{e}-02$ & 2.35 \\
$2^{-18}$ & $2^{-6}$ & $2.226 \mathrm{e}-03$ & 1.98 & $1.339 \mathrm{e}-02$ & 2.61 \\
$2^{-18}$ & $2^{-7}$ & $5.645 \mathrm{e}-04$ & 2.13 & $2.198 \mathrm{e}-03$ & 1.96 \\
$2^{-18}$ & $2^{-8}$ & $1.285 \mathrm{e}-04$ & & $5.661 \mathrm{e}-04$ & \\
$2^{-18}$ & $2^{-9}$ & & & & \\
\hline
\end{tabular}


ence scheme (4.6a)-(4.6f) is also convergent not only when $\frac{\tau}{h}=2^{-5}, 2^{-6}, 2^{-7}, 2^{-8}, 2^{-9}$ but also only when $\frac{\tau}{h}=2^{5}, 2^{6}, 2^{7}, 2^{8}, 2^{9}$. This tells us that the condition $\lambda<1$ in Theorem 4.3 may be unnecessary. We conjecture that this condition may be removed for the convergence of the three-level difference scheme (4.6a)-(4.6f). At present, our analytical technique is limited.

\section{Conclusions}

Korteweg-de Vries equation is a spatial third order nonlinear equations. The solution of initial and boundary problem (1.1a)-(1.1c) satisfies energy conservation (2.4). In this paper, we construct two effective finite difference schemes for nonlinear KdV equations. The existence of the both schemes are proved with the different methods. The conservation and convergence of the finite difference schemes are shown by the energy method. The convergence orders are $\mathcal{O}\left(h+\tau^{2}\right)$ in discrete $L_{2}$ norm. In the future, we will try to establish spatial second order finite difference schemes for the problem (1.1a)-(1.1c). We have found this kind difference scheme. The difficulty is how to prove the global convergence of the difference scheme.

Acknowledgements The project is supported by National Natural Science Foundation of China grant number No. 11671081.

\section{References}

[1] G. D. AKRIVIS, Finite difference discretization of the cubic Schrödinger equation, IMA J. Numer. Anal., 13 (1993), pp. 115-124.

[2] A. Samit, N. Panda, C. Michoski And C. Dawson, A hybridized discontinuous Galerkin method for the nonlinear Korteweg-de Vries equation, J. Sci. Comput., 68 (2016), pp. 191-212.

[3] P. Amorim And M. Figueira, Convergence of a finite difference method for the KdV and modified KdV equations with $L^{2}$ data, Math. Numer. Anal., 58 (2013), pp. 23-50.

[4] N. ATOUANI AND K. OMRANI, On the convergence of conservative difference schemes for the 2D generalized Rosenau-Korteweg de Vries equation, Appl. Math. Comput., 250 (2015), pp. 832-847.

[5] J. BoussinesQ, Essai Sur La Théories Des Eaux Courantes, Impr. Nationale, 1877.

[6] E. F BROWDER, Existence and uniqueness theorems for solutions of nonlinear boundary value problems, Proceedings of Symposia in Applied Mathematics, Providence: AMS, 17 (1965), pp. 24-29.

[7] L. Debnacth, Nonlinear Partial Differential Equations for Scientists and Engineers, Birkhauser, Boston, 2005.

[8] K. Djidjeli, W. G. Price And E. H. Twizell et Al., Numerical methods for the solution of the third- and fifth-order disperive Korteweg-de Vries equations, J. Comput. Appl. Math., 58 (1995), pp. 307-336.

[9] V. A. Dougalis AND O. A. Karakashian, On some high order accurate fully discrete Galerkin methods for the Kortweg-de Vries equation, Math. Comput., 45 (1985), pp. 329345. 
[10] B. GarcíA-ARChILla, A supraconvergent scheme for the Korteweg-de Vries equation, Numerische Mathematik, 61 (1992), pp. 291-310.

[11] H. Holden, U. Koley AND N. Risebro, Convergence of a fully discrete finite difference scheme for the Korteweg-de Vries equation, J. Numer. Anal., 35 (2015), pp. 1047-1077.

[12] S. M. Islam, K. Khan AND M. A. AKBAR, Exact solutions of unsteady Korteweg-de Vries and time regularized long wave equations, Springerplus, 4 (2015), pp. 1-11.

[13] K. Kamruzzaman and A. M. Ali, Solving unsteady Korteweg-de Vries equation and its two alternatives, Math. Methods Appl. Sci., 39 (2016), pp. 2752-2760.

[14] D. J KorteWeg AND G. DE VRIEs, On the change of form of long waves advacing in a rectangular canal and on a new type of long stationary waves, Philosophical Magazine Series, 539 (1895), pp. 422-443.

[15] J. LI, H. MA AND Q. SUn, Error analysis for solving the Korteweg-de Vries equation by a Legendre pseudo-spectral method, Numer. Methods Partial Differential Equations, 16 (2000), pp. 513-534.

[16] A. F. Pazoto, M. SEpúlveda And O. V. Villagrán, Uniform stabilization of numerical schemes for the critical generalized Korteweg-de Vries equation with damping, Numerische Mathematik, 116 (2010), pp. 317-356.

[17] F. L. QU AND W. Q. WANG, Alternating segment explicit-implicit scheme for nonlinear third-order KdV equation, Appl. Math. Mech., 28 (2007), pp. 937-980.

[18] A. QuARTERoni AND A. VAlLI, Numerical Approximation of Partial Differential Equations, Springer-Verlag, New York, 1997.

[19] H. Sun AND Z. Z. Sun, On two linearized difference schemes for Burgers equation, Int. J. Comput. Math., 92 (2015), pp. 1160-1179.

[20] Z. Z. Sun, Numerical Methods of Partial Differential Equations, Second Edition, Science Press, Beijing, 2012.

[21] Z. Z. Sun, W. P. Yuan And Z. C. Wen, Numerical Analysis, 3rd edition, Southeast University Press, Nanjing, 2011.

[22] A. C. Vliegenthart, On finite-difference methods for the Korteweg-de Vries equation, J. Eng. Math., 5 (1971), pp. 137-155.

[23] J. A. WALKer, Dynamical Systems and Evolution Equations, Theory and Applications, Plenum Press, New York, 1980.

[24] A. M. WAzwAz, Partial Differential Equations and Solitary Waves Theory, Higher Education Press: Beijing and Springer-Verlag Berlin, 2009

[25] S. B. Wineberg, J. F. MCgrath, E. F. Gabl, L. R. Scott And C. E. Southwell, Implicit spectral methods for wave propagation problem, J. Comput. Phys., 97 (1991), pp. 311336.

[26] J. L. YAN, Q. ZHANG AND Z. Y. ZHANG, New conservative finite volume element schemes for the modified Korteweg-de Vries equation, Math. Methods Appl. Sci., 39 (2016), pp. 5149-5161.

[27] S. ZHU, A scheme with a higher-order discrete invariant for the KdV equation, Appl. Math. Lett., 14 (2001), pp. 17-20. 